\title{
Decentralized Output Sliding-mode Fault-tolerant Control for Heterogeneous Multi-agent Systems
}

\author{
Chun Liu, Bin Jiang, Senior Member, IEEE, Ron J. Patton, Life Fellow, IEEE, \\ and Ke Zhang, Senior Member, IEEE
}

\begin{abstract}
This study proposes a novel decentralized output sliding-mode fault-tolerant control design for heterogeneous multi-agent systems with matched disturbances, unmatched nonlinear interactions, and actuator faults. The respective iteration and iteration-free algorithms in the sliding-mode fault-tolerant control scheme are designed with adaptive upper bounding laws to automatically compensate the matched and unmatched components. Then, a continuous fault-tolerant protocol in the observerbased integral sliding-mode design is developed to guarantee the asymptotic stability of multi-agent systems and the ultimate boundedness of the estimation errors. Simulation results validate the efficiency of the proposed fault-tolerant control algorithm.
\end{abstract}

Index Terms-Decentralized output feedback, sliding-mode fault-tolerant control, integral sliding-mode control, heterogeneous multi-agent systems, actuator faults.

\section{INTRODUCTION}

Multi-agent systems (MASs) have attracted considerable attention in diverse fields, such as aerospace, transportation, wireless networks, and power systems[1], [2]. Heterogeneous multi-agent systems (HMASs) are MASs composed of a large number of non-identical agents that are connected by mechanical interconnections or communication networks[3]-[6]. A decentralized control is equipped with a simpler architecture with local information than a single centralized control and thus is more practical to realize on physical HMASs[7]-[9]. One essential issue of the decentralized control for HMASs involves the interaction of different agents[10]. Thus, remarkable benefits can be obtained by specifying the decentralized control concept for HMASs with interactions and subsequently achieve the satisfactory local performance of each agent and the global property of the overall HMASs.

Faults may occur more frequently in HMASs than in single agents due to the existence of a number of controllers, sensors, and interconnected equipments[11], [12]. Therefore, HMASs are required to operate safely, and the fault-tolerant control (FTC) is regarded an effective approach to guarantee the stability and desired performance of HMASs with unpredicted faults. An active FTC scheme was presented with a high-gain

C. Liu, B. Jiang and K. Zhang are with the College of Automation Engineering, Nanjing University of Aeronautics and Astronautics, Nanjing 210016, China (e-mail: binjiang@ @uaa.edu.cn).

R. J. Patton is with the School of Engineering and Computer Science, University of Hull, Cottingham Road, Hull HU6 7RX, UK.

This work was supported by National Natural Science Foundation of China (61773201, 61622304 and 61673207); Fundamental Research Funds for the Central Universities (NE2014202); Priority Academic Program Development of Jiangsu Higher Education Institutions; Qing Lan Project of Jiangsu Province; Postgraduate Research and Practice Innovation Program of Jiangsu Province (KYCX17_0269); and China Scholarship Council (201706830025). observer for high-order HMASs in the presence of actuator faults and communication disconnections[13]. An FTC design with fault detection and recovery mechanism was developed for stabilization and navigation of heterogeneous multi-agent formations of autonomous aerial and ground robots[14]. However, most existing studies of HMASs in dealing with faults have centralized or distributed configurations. The centralized and distributed schemes are easy to implement, but both rely on information sharing and transmission among agents[15][19]. A decentralized FTC (DFTC) design should be able to automatically compensate the effects of faults without necessarily exchanging information between individual agents[20]. Various DFTC approaches, such as adaptive DFTC[21], [22], fuzzy DFTC[23], [24], and neural network-based DFTC[25], [26], have been introduced by research in recent years. Most of the results are based on either the state[7], [23], [27] or output feedbacks[4], [8], [13], [25]. The literature indicates that the research on local output DFTC for HMASs has received minimal attention thus far. Furthermore, the DFTC protocol in [20] is usually effective for HMASs with weak interactions and small couplings, and more general assumption is needed in this study compared with the strong interconnections[26], [28]. In addition, the existing disturbances and actuator faults in single agents are easy to spread in HMASs. The sliding mode control (SMC) has been widely applied in handling uncertainties and improving the robustness of HMASs[29][31]. A continuous SMC method with an adaptive strategy was designed for second-order nonlinear MASs with actuator faults and disturbances to realize the consensus-tracking objective[30]. An SMC design with a heterogeneous finitetime disturbance observer was proposed for high-order $\mathrm{H}$ MASs to attenuate the effect of uncertainties[31]. On the one hand, most results on SMC for HMASs focused on matched disturbances in the input channel and did not consider the unmatched one, such as flexible joint manipulators and multimachine power systems[9], which may not always satisfy the matching condition[24], [32]. On the other hand, the results on the decentralized output sliding-mode fault-tolerant control (SM-FTC) of coupled nonlinear HMASs with matched disturbances, unmatched nonlinear interactions, and actuator faults are dearth, hence the motivation of the present investigation.

To tackle the above mentioned difficulty, the decentralized output SM-FTC scheme for HMASs is designed with adaptive upper bounding laws. The matched disturbances, unmatched interactions, and actuator faults are effectively compensated, and then the robust performance of the SM-FTC strategy is further achieved by rigorously performing a stability analysis 
of HMASs and conducting a reachability analysis of the SMC motion. To the best of our knowledge, integral SMC can be adopted in FTC schemes for various complex MASs[33]-[35]. Compared with the conventional SMC method that partitions the state space into matched and unmatched parts[29], [36], the matched disturbances and actuator faults are compensated in the integral SMC strategy[37]. An integral SMC scheme was presented for HMASs composed of quadrotors and twowheeled mobile robots associated with model uncertainties and external disturbances to reach a consensus[34]. Actuator faults and external disturbances/model uncertainties in a group of nonlinear systems were tolerated by using the integral SMC technique[35]. More importantly, there are few literature on the subject of observer-based consensus for MASs[38], [39]. The distributed pinning observer[40] and the finite-time observer[41] in the consensus control strategy are designed to estimate the state information of each single agent. In this case, further introducing the observer-based integral SMC into the decentralized output SM-FTC strategy for HMASs can lead to complex research and analysis.

The major contributions of this study can be summarized as follows. (i) This study is the first trial to consider the output DFTC problem for HMASs with actuator faults and nonlinear interactions, which contain unmatched components, in contrast with the existing work that focuses mostly on matched model uncertainties and external disturbances[26], [30], [34]. To overcome this difficulty, an augmented dynamic configuration is constructed with the dynamic compensator to satisfy the Kimura-Davison condition[42] and the decentralized output SM-FTC technique is introduced. (ii) In comparison with the adaptive bounds[21], prescribed performance bounds[22], and Lipschitz boundedness of the disturbances, model uncertainties and nonlinearities[13], [19], [23], [35], the adaptive law is used in this study to relax the known upper bound assumptions of the matched disturbances and actuator faults. (iii) Two multistep algorithms based on the extended linear matrix inequality (LMI) characterization are given to reduce the conservativeness of the solution of the nonlinear matrix inequality with non-convex algebraic constraints by implementing iteration and iteration-free strategies.

The remainder of this study is organized as follows. Section II introduces the system formulation. Section III is devoted to the SMC design with the stability analysis of HMASs and reachability analysis of the SMC motion. The iteration and iteration-free algorithms are further illustrated. The observerbased integral SMC design is proposed in Section IV to guarantee the ultimately uniformly boundedness of the estimation errors. Simulations in Section V validate the efficiency of the proposed algorithm. Finally, conclusions follow in Section VI. The symbol $\operatorname{sgn}(\cdot)$ denotes the sign function, the vector $x=\operatorname{col}\left(x_{i}\right)=\left[x_{1}^{T}, \cdots, x_{N}^{T}\right]^{T}$, and the matrix $Y=\operatorname{diag}\left(Y_{i}\right)$ is of diagonal form with elements of matrices $Y_{i}, i=1, \cdots, N \cdot \operatorname{He}(X)=X+X^{T}$ and $\star$ denotes the symmetric part of the specific matrix.

\section{SYSTEM FORMULATION}

Consider a group of $N$ agents in the presence of matched disturbances, unmatched nonlinear interactions, and actuator faults. The $i$-th agent of HMASs $(i=1, \cdots, N)$ is given as

$$
\begin{aligned}
\dot{x}_{i}(t)= & A_{i} x_{i}(t)+B_{i}\left(u_{i}(t)+d_{i}\left(x_{i}, u_{i}, t\right)\right) \\
& +F_{i} f_{i}(t)+\xi_{i}(x, t) \\
y_{i}(t)= & C_{i} x_{i}(t)
\end{aligned}
$$

where $x_{i} \in R^{n_{i}}, u_{i} \in R^{m_{i}}$ and $y_{i} \in R^{p_{i}}$ are the system state, input and output vectors respectively, $d_{i}\left(x_{i}, u_{i}, t\right) \in R^{m_{i}}$ denotes the matched disturbance, $f_{i}(t) \in R^{q_{i}}$ denotes the actuator fault and $\xi_{i}(x, t) \in R^{n_{i}}$ denotes the nonlinear interaction in HMASs with the global state vector $x=\operatorname{col}\left(x_{i}\right)$. Matrices $A_{i}, B_{i}, F_{i}$ and $C_{i}$ are known with compatible dimensions and $m_{i} \leq p_{i} \leq n_{i}$ is satisfied.

Assumption 2.1: (i) The pairs $\left(A_{i}, B_{i}\right)$ and $\left(A_{i}, C_{i}\right)$ are controllable and observable, respectively. (ii) The actuator fault $f_{i}(t)$ is satisfied with $\operatorname{rank}\left(B_{i}, F_{i}\right)=\operatorname{rank}\left(B_{i}\right)$ and $\operatorname{rank}\left(C_{i} F_{i}\right)=\operatorname{rank}\left(F_{i}\right)$, and it belongs to $\mathcal{L}_{2}[0, \infty)$, i.e., $\left\|f_{i}(t)\right\| \leq \bar{f}_{i}$ with the unknown upper bound $\bar{f}_{i}$. (iii) The matched disturbance $d_{i}\left(x_{i}, u_{i}, t\right)$ belongs to $\mathcal{L}_{2}[0, \infty)$, i.e., $\left\|d_{i}\left(x_{i}, u_{i}, t\right)\right\| \leq \bar{d}_{i}$ with the unknown upper bound $\bar{d}_{i}$.

Assumption 2.2: The nonlinear interaction term $\xi_{i}(x, t)$ in HMASs satisfies with the quadratic constraint, i.e., $\xi_{i}^{T}(x, t) \xi_{i}(x, t) \leq \alpha_{i}^{2} x^{T} E_{i}^{T} E_{i} x$, where $E_{i}$ is a known constant matrix and $\alpha_{i}$ is a known upper bound scalar.

Remark 2.1: Assumption 2.1 provides the controllable and observable conditions for the described HMASs and guarantees the actuator fault $f_{i}(t)$ to be constrained in a given compensation range. The interaction term $\xi_{i}(x, t)$ in Assumption 2.2 can be described as the physical interconnections, e.g. the transmission links of smart grids[1] and the interconnections of multi-machine power systems[9], [36]. Define $\xi(x, t)=$ $\operatorname{col}\left(\xi_{i}(x, t)\right)$, and it follows that the overall interaction term satisfies $\xi^{T}(x, t) \xi(x, t) \leq x^{T}\left(\sum_{i=1}^{N} \alpha_{i}^{2} E_{i}^{T} E_{i}\right) x=x^{T} E^{T} E x$ with $E=\left[\alpha_{1} E_{1}^{T}, \cdots, \alpha_{N} E_{N}^{T}\right]^{T}$.

Remark 2.2: In comparison with the known smooth and bounded interactions[28], the known upper bounds of the actuator faults[24], [26] and external disturbances[16], and the unknown but locally Lipschitz nonlinearities[13], [19], [23], the proposed assumptions in this study are more general with adaptively approximating a surrogate of the upper bounds. By contrast, the previous works on actuator faults have been combined with interconnection delays[21], [22], unmeasured states[23], and unstructured uncertainties[25], [26].

Lemma 2.1[43]: Consider the following inequality with a symmetric matrix $\Omega \in R^{l \times l}$ and matrices $S$ and $H$ of the column dimension $l$ :

$$
\Omega+S^{T} X H+H^{T} X^{T} S<0
$$

and the inequality (2) has a solution of matrix $X$ of compatible dimensions if and only if $N_{S}^{T} \Omega N_{S}<0$ and $N_{H}^{T} \Omega N_{H}<0$ are satisfied, where $N_{S}$ and $N_{H}$ represent the column form bases of the null spaces of $S$ and $H$, respectively. Furthermore, on the basis of Finsler's Lemma, the inequalities $N_{S}^{T} \Omega N_{S}<0$ and $N_{H}^{T} \Omega N_{H}<0$ hold if and only if there exist scalars $\mu_{S}$ and $\mu_{H}$ such that $\Omega-\mu_{S} S^{T} S<0$ and $\Omega-\mu_{H} H^{T} H<0$.

\section{SMC DESIGN AND STABILITY ANALYSIS}

A dynamic compensator of the appropriate dimension is used in the output feedback SMC design to satisfy the Kimura- 
Davison condition[42]. On the basis of the available output information, the dynamic compensator is given by

$$
\dot{\bar{x}}_{i}(t)=\bar{A}_{i} \bar{x}_{i}(t)+\bar{B}_{i} y_{i}(t), \bar{x}_{i}\left(t_{0}\right)=\bar{x}_{i 0}
$$

and the decentralized control input is given associated with the dynamic compensator (3) as follows

$$
u_{i}(t)=\bar{C}_{i} \bar{x}_{i}(t)+\bar{D}_{i} y_{i}(t)+v_{i}(t)
$$

where $\bar{x}_{i} \in R^{r_{i}}$ denotes the state of the compensator and $v_{i} \in R^{m_{i}}$ denotes the nonlinear SM-FTC switching function to compensate for the effects of matched disturbances and actuator faults. Matrices $\bar{A}_{i}, \bar{B}_{i}, \bar{C}_{i}$ and $\bar{D}_{i}$ are system gains with compatible dimensions to be designed.

Define the augmented vectors $\tilde{x}_{i}=\left[\begin{array}{ll}x_{i}^{T} & \bar{x}_{i}^{T}\end{array}\right]^{T}$ and $\tilde{y}_{i}=$ $\left[\begin{array}{ll}y_{i}^{T} & \bar{x}_{i}^{T}\end{array}\right]^{T}$. The $i$-th augmented dynamics of the combined HMASs and the dynamic compensator are described as

$$
\begin{aligned}
\dot{\tilde{x}}_{i}(t)= & \left(\tilde{A}_{i}+\tilde{B}_{i} K_{i} \tilde{C}_{i}\right) \tilde{x}_{i}(t)+\tilde{F}_{i} f_{i}(t) \\
& +\tilde{B}_{i 0}\left(d_{i}\left(x_{i}, u_{i}, t\right)+v_{i}(t)\right)+\tilde{G}_{i} \xi_{i}(x, t) \\
\tilde{y}_{i}(t)= & \tilde{C}_{i} \tilde{x}_{i}(t)
\end{aligned}
$$

where

$$
\begin{aligned}
& \tilde{A}_{i}=\left[\begin{array}{cc}
A_{i} & 0 \\
0 & 0
\end{array}\right], \tilde{B}_{i}=\left[\begin{array}{cc}
B_{i} & 0 \\
0 & I_{r_{i}}
\end{array}\right], K_{i}=\left[\begin{array}{cc}
\bar{D}_{i} & \bar{C}_{i} \\
\bar{B}_{i} & \bar{A}_{i}
\end{array}\right] \\
& \tilde{C}_{i}=\left[\begin{array}{cc}
C_{i} & 0 \\
0 & I_{r_{i}}
\end{array}\right], \tilde{B}_{i 0}=\left[\begin{array}{c}
B_{i} \\
0
\end{array}\right], \tilde{F}_{i}=\left[\begin{array}{c}
F_{i} \\
0
\end{array}\right], \tilde{G}_{i}=\left[\begin{array}{c}
I_{n_{i}} \\
0
\end{array}\right]
\end{aligned}
$$

Then, the augmented HMASs can be written as

$$
\begin{aligned}
\dot{\tilde{x}}(t)= & (\tilde{A}+\tilde{B} K \tilde{C}) \tilde{x}(t)+\tilde{F} f(t) \\
& +\tilde{B}_{0}(d(x, u, t)+v(t))+\tilde{G} \xi(x, t) \\
\tilde{y}(t)= & \tilde{C} \tilde{x}(t)
\end{aligned}
$$

where $\tilde{x}=\operatorname{col}\left(\tilde{x}_{i}\right) \in R^{n+r}, \tilde{y}=\operatorname{col}\left(\tilde{y}_{i}\right) \in R^{p+r}, d=$ $\operatorname{col}\left(d_{i}\right) \in R^{m}, v=\operatorname{col}\left(v_{i}\right) \in R^{m}, f=\operatorname{col}\left(f_{i}\right) \in R^{q}$. Matrices $\tilde{A}, \tilde{B}, \tilde{B}_{0}, K, \tilde{C}, \tilde{F}$ and $\tilde{G}$ are of diagonal forms with elements of the respective matrices $\tilde{A}_{i}, \tilde{B}_{i}, \tilde{B}_{i 0}, K_{i}, \tilde{C}_{i}, \tilde{F}_{i}$ and $\tilde{G}_{i}$. Scalars $n=\sum_{i=1}^{N} n_{i}, m=\sum_{i=1}^{N} m_{i}, q=\sum_{i=1}^{N} q_{i}, p=$ $\sum_{i=1}^{N} p_{i}$ and $r=\sum_{i=1}^{\bar{N}} r_{i}$.

The nonlinear interaction term $\tilde{G} \xi(x, u, t)$ in (6) can be written in the following form with $\tilde{E}=\left[\alpha_{1} \tilde{E}_{1}^{T}, \cdots, \alpha_{N} \tilde{E}_{N}^{T}\right]^{T}$.

$$
\begin{aligned}
\xi^{T} \tilde{G}^{T} \tilde{G} \xi & \leq \tilde{x}^{T}\left(\sum_{i=1}^{N} \alpha_{i}^{2}\left[\begin{array}{cc}
E_{i} & 0 \\
0 & 0
\end{array}\right]^{T}\left[\begin{array}{cc}
E_{i} & 0 \\
0 & 0
\end{array}\right]\right) \tilde{x} \\
& =\tilde{x}^{T}\left(\sum_{i=1}^{N} \alpha_{i}^{2} \tilde{E}_{i}^{T} \tilde{E}_{i}\right) \tilde{x}=\tilde{x}^{T} \tilde{E}^{T} \tilde{E} \tilde{x}
\end{aligned}
$$

The SMC function for the $i$-th augmented HMASs on the basis of the newly available output information $\tilde{y}_{i}=\left[\begin{array}{ll}y_{i}^{T} & \bar{x}_{i}^{T}\end{array}\right]^{T}$ is designed as

$$
\sigma_{i}\left(\bar{x}_{i}, y_{i}, t\right)=T_{i} \tilde{y}_{i}=\left[T_{i 1} T_{i 2}\right] \tilde{C}_{i} \tilde{x}_{i}=T_{i 1} y_{i}+T_{i 2} \bar{x}_{i}
$$

where $T_{i 1} \in R^{m_{i} \times p_{i}}$ and $T_{i 2} \in R^{m_{i} \times r_{i}}$ are matrices to be designed. Furthermore, define the upper bound estimation errors $e_{d_{i}}=\hat{\bar{d}}_{i}-\bar{d}_{i}$ and $e_{f_{i}}=\hat{\bar{f}}_{i}-\bar{f}_{i}$, where $\hat{\bar{d}}_{i}$ and $\hat{\bar{f}}_{i}$ are the estimations of the unknown upper bounds $\bar{d}_{i}$ and $\bar{f}_{i}$, respectively. Here, the adaptive algorithms for the estimated upper bound parameters $\hat{\bar{d}}_{i}$ and $\hat{\bar{f}}_{i}$ are designed as

$$
\dot{\overline{\hat{d}}}_{i}=\left\|B_{i}\right\|\left\|\tilde{y}_{i}\right\|, i=1, \cdots, N
$$

$$
\dot{\overline{f_{i}}}=\left\|F_{i}\right\|\left\|\tilde{y}_{i}\right\|, i=1, \cdots, N
$$

Remark 3.1. If the so-called Kimura-Davison condition[42] is not satisfied, it is verified that it is difficult to propose a static output feedback SMC scheme. In this case, a sufficient condition to solve this problem is adding an extra compensator (3), (4) (i.e. a particular subsystem with the relative dimensions $\bar{x}_{i} \in R^{r_{i}}$ ), thus, providing additional degrees of freedom.

Here, the following theorem is given to achieve the stability of the augmented HMASs and the insensitivity to the matched disturbances and actuator faults.

Theorem 3.1. Consider the augmented HMASs (6) and the decentralized control input (4), the overall dynamic system is quadratically stable and the matched disturbances and actuator faults can be compensated with the adaptive algorithms (9), (10) and the following SM-FTC switching function $v_{i}$ if there exist matrices $W_{1 i} \in R^{z_{i} \times z_{i}}, W_{2 i} \in R^{\left(p_{i}+r_{i}\right) \times\left(p_{i}+r_{i}\right)}$ and matrix $P=\operatorname{diag}\left(P_{i}\right)$ with each symmetric positive-definite matrix $P_{i} \in R^{\left(n_{i}+r_{i}\right) \times\left(n_{i}+r_{i}\right)}$ such that

$$
\tilde{\mathcal{B}}_{i 0} W_{1 i} \tilde{\mathcal{B}}_{i 0}^{T}+\tilde{C}_{i}^{T} W_{2 i} \tilde{C}_{i}>0
$$

$$
\operatorname{He}(P \tilde{A}+P \tilde{B} K \tilde{C})+P P+\tilde{E}^{T} \tilde{E}<0
$$

where $\tilde{\mathcal{B}}_{i 0} \in R^{\left(n_{i}+r_{i}\right) \times z_{i}}$ is the orthogonal matrix of $\tilde{B}_{i 0}$ satisfying with $\tilde{\mathcal{B}}_{i 0}^{T} \tilde{B}_{i 0}=0$. The SM-FTC switching function $v_{i}$ in the decentralized control input (4) is designed as

$$
\begin{aligned}
v_{i}(t)= & -\left\|\left(T_{i} \tilde{C}_{i}\right)^{\dagger}\right\|\left(\left\|P_{i}\right\|\left(\left\|B_{i}\right\| \hat{\bar{d}}_{i}+\left\|F_{i}\right\| \hat{\bar{f}}_{i}\right)\right. \\
& \left.+\eta_{i}\right) \operatorname{sgn}\left(\sigma_{i}\left(\bar{x}_{i}, y_{i}, t\right)\right)
\end{aligned}
$$

where $\left(T_{i} \tilde{C}_{i}\right)^{\dagger}=\left(\left(T_{i} \tilde{C}_{i}\right)^{T}\left(T_{i} \tilde{C}_{i}\right)\right)^{-1}\left(T_{i} \tilde{C}_{i}\right)^{T}$ and $\eta_{i}$ is a positive scalar. The sign function $\operatorname{sgn}\left(\sigma_{i}\left(\bar{x}_{i}, y_{i}, t\right)\right)=0$ when $\sigma_{i}\left(\bar{x}_{i}, y_{i}, t\right)=0$ is satisfied, which implies that each SMC function has been reached. Then, the gain matrices of the nonlinear SM-FTC switching function (13) are derived as

$$
P_{i}=\tilde{\mathcal{B}}_{i 0} W_{1 i} \tilde{\mathcal{B}}_{i 0}^{T}+\tilde{C}_{i}^{T} W_{2 i} \tilde{C}_{i}, T_{i}=\tilde{B}_{i 0}^{T} \tilde{C}_{i}^{T} W_{2 i}^{T}
$$

Proof. Since $P_{i}=\tilde{\mathcal{B}}_{i 0} W_{1 i} \tilde{\mathcal{B}}_{i 0}^{T}+\tilde{C}_{i}^{T} W_{2 i} \tilde{C}_{i}$ in (14) is a symmetric positive-definite matrix, a Lyapunov function $V_{\tilde{x}}$ for the augmented HMASs (6) is considered as follows

$$
V_{\tilde{x}}=\tilde{x}^{T} P \tilde{x}+\sum_{i=1}^{N}\left\|P_{i}\right\|\left\|\tilde{C}_{i}\right\|^{-1}\left(e_{d i}^{2}+e_{f i}^{2}\right)
$$

The time derivative of $V_{\tilde{x}}$ in (15) is obtained as

$$
\begin{aligned}
\dot{V}_{\tilde{x}}= & \tilde{x}^{T}\left(P \tilde{A}+\tilde{A}^{T} P+P \tilde{B} K \tilde{C}+\tilde{C}^{T} K^{T} \tilde{B}^{T} P\right) \tilde{x} \\
& +2 \tilde{x}^{T} P \tilde{G} \xi+\sum_{i=1}^{N} 2 \tilde{x}_{i}^{T} P_{i} \tilde{B}_{i 0}\left(d_{i}+v_{i}\right) \\
& +\sum_{i=1}^{N} 2 \tilde{x}_{i}^{T} P_{i} \tilde{F}_{i} f_{i} \\
& +\sum_{i=1}^{N} 2\left\|P_{i}\right\|\left\|\tilde{C}_{i}\right\|-1\left\|\tilde{y}_{i}\right\|\left(\left\|B_{i}\right\| e_{d i}+\left\|F_{i}\right\| e_{f i}\right) \\
\leq & \tilde{x}^{T}\left(P \tilde{A}+\tilde{A}^{T} P+P \tilde{B} K \tilde{C}+\tilde{C}^{T} K^{T} \tilde{B}^{T} P\right. \\
& \left.+P P+\tilde{E}^{T} \tilde{E}\right) \tilde{x}+\sum_{i=1}^{N} 2 \tilde{x}_{i}^{T} P_{i} \tilde{B}_{i 0} v_{i} \\
& +\sum_{i=1}^{N}\left(2\left\|\tilde{x}_{i}\right\|\left\|P_{i}\right\|\left(\left\|B_{i}\right\| \bar{d}_{i}+\left\|F_{i}\right\| \bar{f}_{i}\right)\right) \\
& +\sum_{i=1}^{N} 2\left\|P_{i}\right\|\left\|\tilde{x}_{i}\right\|\left(\left\|B_{i}\right\| e_{d i}+\left\|F_{i}\right\| e_{f i}\right)
\end{aligned}
$$

Since $P_{i}=\tilde{\mathcal{B}}_{i 0} W_{1 i} \tilde{\mathcal{B}}_{i 0}^{T}+\tilde{C}_{i}^{T} W_{2 i} \tilde{C}_{i}$ and $T_{i}=\tilde{B}_{i 0}^{T} \tilde{C}_{i}^{T} W_{2 i}^{T}$, it is derived as $P_{i} \tilde{B}_{i 0}=\tilde{C}_{i}^{T} T_{i}^{T}$. Then, it follows that

$$
\begin{aligned}
\dot{V}_{\tilde{x}} \leq & \tilde{x}^{T}\left(P \tilde{A}+\tilde{A}^{T} P+P \tilde{B} K \tilde{C}+\tilde{C}^{T} K^{T} \tilde{B}^{T} P\right. \\
& \left.+P P+\tilde{E}^{T} \tilde{E}\right) \tilde{x}-\sum_{i=1}^{N} 2\left\|\tilde{x}_{i}\right\| \eta_{i}
\end{aligned}
$$


It is obtained that $\dot{V}_{\tilde{x}}<0$ when the inequality in (12) and $\eta_{i}>0$ are satisfied. This completes the proof.

Notably, the inequality in (12) is not convex and thus cannot be solved with the LMI toolbox. Here, the following corollary is given with iteration to solve the non-convex constraints.

Corollary 3.1. Given the pre-designed symmetric matrix $\tilde{P}$, scalars $\alpha_{i}$, positive scalars $\mu_{\tilde{C}}$ and $\mu_{W}$, and matrices $W_{1}=$ $\operatorname{diag}\left(W_{1 i}\right), W_{2}=\operatorname{diag}\left(W_{2 i}\right)$, the inequality in (12) is solvable for the symmetric positive-definite matrix $P=\tilde{\mathcal{B}}_{0} W_{1} \tilde{\mathcal{B}}_{0}^{T}+$ $\tilde{C}^{T} W_{2} \tilde{C}>0$ with a diagonal matrix $\tilde{\mathcal{B}}_{0}=\operatorname{diag}\left(\tilde{\mathcal{B}}_{i 0}\right)$ if the following minimization problem is solvable.

$$
\begin{gathered}
{\left[\begin{array}{ccccc}
\Omega_{1} & P & \tilde{E}_{1}^{T} & \cdots & \tilde{E}_{N}^{T} \\
\star & -I_{n+r} & 0 & \cdots & 0 \\
\star & \star & -\alpha_{1}^{-2} I & \cdots & 0 \\
\vdots & \vdots & \vdots & \ddots & \vdots \\
\star & \star & \star & \cdots & -\alpha_{N}^{-2} I
\end{array}\right]<0 \quad \text { (18) }} \\
{\left[\begin{array}{cccccc}
\Omega_{2} & P & \tilde{P} \tilde{B} & \tilde{E}_{1}^{T} & \cdots & \tilde{E}_{N}^{T} \\
\star & -I_{n+r} & 0 & 0 & \cdots & 0 \\
\star & \star & -\mu_{W} I_{m+r} & 0 & \cdots & 0 \\
\star & \star & \star & -\alpha_{1}^{-2} I & \cdots & 0 \\
\vdots & \vdots & \vdots & \vdots & \ddots & \vdots \\
\star & \star & \star & \star & \cdots & -\alpha_{N}^{-2} I
\end{array}\right]<0}
\end{gathered}
$$

where $\Omega_{1}=\operatorname{He}(P \tilde{A})-\mu_{\tilde{C}} \tilde{C}^{T} \tilde{C}$ and $\Omega_{2}=\operatorname{He}(P(\tilde{A}+\tilde{B} \tilde{K}))$ with the derived matrix $\tilde{K}=-\tilde{B}^{T} \tilde{P}$.

Proof. According to Lemma 2.1, it is easy to check that the inequality in (12) is in the form of that in (2). Define $W=\tilde{B}^{T} P$, and the inequality in (12) is solvable for matrix $K$ if and only if there exists a symmetric positive-definite matrix $P$ such that

$$
\begin{aligned}
& N_{\tilde{\tilde{C}}}^{T}\left(P \tilde{A}+\tilde{A}^{T} P+P P+\tilde{E}^{T} \tilde{E}\right) N_{\tilde{C}}<0 \\
& N_{W}^{T}\left(P \tilde{A}+\tilde{A}^{T} P+P P+\tilde{E}^{T} \tilde{E}\right) N_{W}<0
\end{aligned}
$$

where $N_{\tilde{C}}$ and $N_{W}$ denote the column form bases of the null spaces of $\tilde{C}$ and $W$, respectively. Then, the inequalities in (20) are solvable by using Finsler's Lemma if the following forms are satisfied with positive scalars $\mu_{\tilde{C}}$ and $\mu_{W}$.

$$
\begin{gathered}
P \tilde{A}+\tilde{A}^{T} P+P P+\tilde{E}^{T} \tilde{E}-\mu_{\tilde{C}} \tilde{C}^{T} \tilde{C}<0 \\
P \tilde{A}+\tilde{A}^{T} P+P P+\tilde{E}^{T} \tilde{E}-\mu_{W} P \tilde{B} \tilde{B}^{T} P<0
\end{gathered}
$$

The existing condition, i.e., $\mu_{W} P \tilde{B} \tilde{B}^{T} P>0$, makes the inequality in (22) non-convex, which needs to be further dealt with. Furthermore, the inequality in (19) is equivalent to the following form with $\tilde{K}=-\tilde{B}^{T} \tilde{P}$.

$$
\begin{aligned}
& P \tilde{A}+\tilde{A}^{T} P-P \tilde{B} \tilde{B}^{T} \tilde{P}-\tilde{P} \tilde{B} \tilde{B}^{T} P+P P \\
& +\tilde{E}^{T} \tilde{E}+\mu_{W}^{-1} \tilde{P} \tilde{B} \tilde{B}^{T} \tilde{P}<0 \\
\Rightarrow & P \tilde{A}+\tilde{A}^{T} P-\mu_{W} P \tilde{B} \tilde{B}^{T} P+P P+\tilde{E}^{T} \tilde{E}<0
\end{aligned}
$$

Thus, the inequality in (19) is equivalent to that in (22). Meanwhile, the formulation in (18) is equivalent to that in (21). The solvable LMI formulations in (18), (19) imply that there exists a solution of matrix $P$ satisfying the forms in (21), (22). Hence, the inequality in (12) is solvable by using Lemma 2.1 and this completes the proof.

The following algorithm is given with the iteration strategy.

\section{Algorithm 3.1.}

1) Define $\tilde{A}_{s}=\tilde{A}+s I_{n+r}$, where $s$ is the pre-designed degree of stability. Then, solve the following algebraic Riccati equation and derive the symmetric matrix $\tilde{P}$ :

$$
\tilde{P} \tilde{A}_{s}+\tilde{A}_{s}^{T} \tilde{P}-\tilde{P} \tilde{B} \tilde{B}^{T} \tilde{P}+\varepsilon I_{n+r}=0
$$

where $\varepsilon>0$ is arbitrarily small.

2) Set $\tilde{K}=-\tilde{B}^{T} \tilde{P}$ with the derived matrix $\tilde{P}$ in step 1 .

3) Solve the LMI minimization problem in (18), (19) and derive the symmetric matrix $P$.

4) Fix $P=\tilde{\mathcal{B}}_{0} W_{1} \tilde{\mathcal{B}}_{0}^{T}+\tilde{C}^{T} W_{2} \tilde{C}$ and derive the matrices $W_{1}$ and $W_{2}$. Solve the following LMI minimization problem and derive the matrix $K$.

$$
\left[\begin{array}{ccccc}
\Omega_{3} & P & \tilde{E}_{1}^{T} & \cdots & \tilde{E}_{N}^{T} \\
\star & -I_{n+r} & 0 & \cdots & 0 \\
\star & \star & -\alpha_{1}^{-2} I_{n+r} & \cdots & 0 \\
\vdots & \vdots & \vdots & \ddots & \vdots \\
\star & \star & \star & \cdots & -\alpha_{N}^{-2} I_{n+r}
\end{array}\right]<0
$$

where $\Omega_{3}=\operatorname{He}(P(\tilde{A}+\tilde{B} K \tilde{C}))$. Then, the dynamic compensator matrix $K=\operatorname{diag}\left(K_{i}\right)$ and the matrix in the SMC function (8) is derived as $T_{i}=\tilde{B}_{i 0}^{T} \tilde{C}_{i}^{T} W_{2 i}^{T}$.

Furthermore, the matrices $P$ and $K$ in (12) can be decoupled without iteration and the following corollary is given to reduce the space complexity of the LMI formulation.

Corollary 3.2. Given scalars $\alpha_{i}$, and matrices $W_{1}=$ $\operatorname{diag}\left(W_{1 i}\right), W_{2}=\operatorname{diag}\left(W_{2 i}\right)$, the inequality in (12) is solvable for the symmetric positive-definite matrices $\mathcal{P}=\operatorname{diag}\left(P_{i 11}\right)$ and $\mathcal{Q}=\operatorname{diag}\left(Q_{i 11}\right)$ with $P_{i 11}=\mathcal{B}_{i} W_{1 i} \mathcal{B}_{i}^{T}+C_{i}^{T} W_{2 i} C_{i}$ if the following minimization problem is solvable.

$$
\begin{gathered}
{\left[\begin{array}{ccccc}
\Omega_{4} & N_{C}^{T} \mathcal{P} & N_{C}^{T} E_{1}^{T} & \cdots & N_{C}^{T} E_{N}^{T} \\
\star & -I_{n} & 0 & \cdots & 0 \\
\star & \star & -\alpha_{1}^{-2} I_{n} & \cdots & 0 \\
\vdots & \vdots & \vdots & \ddots & \vdots \\
\star & \star & \star & \cdots & -\alpha_{N}^{-2} I_{n}
\end{array}\right]<0} \\
{\left[\begin{array}{cccc}
\Omega_{5} & N_{B}^{T} \mathcal{Q} E_{1}^{T} & \cdots & N_{B}^{T} \mathcal{Q} E_{N}^{T} \\
\star & -\alpha_{1}^{-2} I_{n} & \cdots & 0 \\
\vdots & \vdots & \ddots & \vdots \\
\star & \star & \cdots & -\alpha_{N}^{-2} I_{n}
\end{array}\right]<0} \\
{\left[\begin{array}{ccc}
\mathcal{B} W_{1} \mathcal{B}^{T}+C^{T} W_{2} C & I_{n} \\
\star & & \mathcal{Q}
\end{array}\right]>0}
\end{gathered}
$$

where $\Omega_{4}=N_{C}^{T}(\operatorname{He}(\mathcal{P} A)) N_{C}, \Omega_{5}=N_{B}^{T}\left(\operatorname{He}(A \mathcal{Q})+I_{n}\right) N_{B}$. Matrices $N_{C}$ and $N_{B}$ denote the column form bases of the null spaces of $C$ and $B$, respectively. Matrix $\mathcal{B}=\operatorname{diag}\left(\mathcal{B}_{i}\right)$ and its element $\mathcal{B}_{i} \in R^{n_{i} \times z_{i}}$ is the orthogonal matrix of matrix $B_{i}$ satisfying with $\mathcal{B}_{i}^{T} B_{i}=0$. Matrices $C$ and $B$ are of diagonal forms with elements of matrices $C_{i}$ and $B_{i}$, respectively.

Proof. Define the symmetric positive-definite matrices $P=$ $\operatorname{diag}\left(P_{i}\right)$ and $Q=\operatorname{diag}\left(Q_{i}\right)=P^{-1}$. According to Lemma 2.1 , the necessary and sufficient condition of the feasible solution in (12) is that matrices $P$ and $Q$ are satisfied with

$$
\begin{aligned}
& N_{\tilde{C}}^{T}\left(P \tilde{A}+\tilde{A}^{T} P+P P+\tilde{E}^{T} \tilde{E}\right) N_{\tilde{C}}<0 \\
& N_{\tilde{B}}^{T}\left(\tilde{A} Q+Q \tilde{A}^{T}+I_{n+r}+Q \tilde{E}^{T} \tilde{E} Q\right) N_{\tilde{B}}<0
\end{aligned}
$$


Partition every $P_{i}$ and its inverse matrix $Q_{i}$ as

$$
P_{i}=\left[\begin{array}{cc}
P_{i 11} & P_{i 12} \\
\star & P_{i 22}
\end{array}\right], Q_{i}=\left[\begin{array}{cc}
Q_{i 11} & Q_{i 12} \\
\star & Q_{i 22}
\end{array}\right]
$$

Meanwhile, the following compact is derived on the basis of the matrix inversion lemma.

$$
P_{i}^{-1}=\left[\begin{array}{cc}
\tilde{P}_{i 11} & -\tilde{P}_{i 11} P_{i 12} P_{i 22}^{-1} \\
\star & P_{i 22}^{-1}+P_{i 22}^{-1} P_{i 12}^{T} \tilde{P}_{i 11} P_{i 12} P_{i 22}^{-1}
\end{array}\right]
$$

where $\tilde{P}_{i 11}=\left(P_{i 11}-P_{i 12} P_{i 22}^{-1} P_{i 12}^{T}\right)^{-1}$. Then, it is derived as $P_{i 11}-Q_{i 11}^{-1}=P_{i 12} P_{i 22}^{-1} P_{i 12}^{T}>0$, i.e., $P_{i 11}>Q_{i 11}^{-1}$ and $\mathcal{B}_{i} W_{1 i} \mathcal{B}_{i}^{T}+C_{i}^{T} W_{2 i} C_{i}>Q_{i 11}^{-1}$. The following form is then obtained and is equivalent to the formulation in (28).

$$
\mathcal{B} W_{1} \mathcal{B}^{T}+C^{T} W_{2} C>\mathcal{Q}^{-1}
$$

Select the first row and column in (29), and it is derived as

$$
\begin{aligned}
& N_{C}^{T}\left(\mathcal{P} A+A^{T} \mathcal{P}+\mathcal{P} \mathcal{P}+E^{T} E\right) N_{C}<0 \\
& N_{B}^{T}\left(A \mathcal{Q}+\mathcal{Q} A^{T}+I_{n}+\mathcal{Q} E^{T} E \mathcal{Q}\right) N_{B}<0
\end{aligned}
$$

where $\mathcal{P}=\operatorname{diag}\left(P_{i 11}\right)$ and $\mathcal{Q}=\operatorname{diag}\left(Q_{i 11}\right)$.

The inequalities in (33) are equivalent to that in (26), (27). The solvable LMI formulations in (26)-(28) imply that there exists a solution of matrices $\mathcal{P}, \mathcal{Q}, W_{1}$ and $W_{2}$ satisfying the forms in (32), (33). Thus, the inequality in (12) is solvable by using the following algorithm with an iteration-free strategy and this completes the proof.

\section{Algorithm 3.2.}

1) Solve the LMI minimization problem in (26)-(28) and derive the matrices $\mathcal{P}, \mathcal{Q}, W_{1}$ and $W_{2}$.

2) Define $P_{i 22}=I_{r_{i}}$ and $\mathcal{M}_{i}=P_{i 12} P_{i 12}^{T}$. Then, $P_{i 11}-$ $Q_{i 11}^{-1}=\mathcal{M}_{i}$ is obtained with the derived matrices $\mathcal{P}$ and $\mathcal{Q}$ in step 1 . Then, the following form is obtained with the diagonal decomposition method.

$$
\mathcal{B}_{i} W_{1 i} \mathcal{B}_{i}^{T}+C_{i}^{T} W_{2 i} C_{i}-Q_{i 11}^{-1}=V_{i} \lambda_{i} V_{i}^{T}
$$

where $\lambda_{i}=\operatorname{diag}\left(\lambda_{i, 1}, \cdots, \lambda_{i, n_{i}}\right)$ and $V_{i}$ denote the respective eigenvalues diagonal matrix and eigenvector matrix of matrix $\mathcal{M}_{i}$. Furthermore, define $\tilde{\lambda}_{i}=$ $\operatorname{diag}\left(\sqrt{\lambda_{i, 1}}, \cdots, \sqrt{\lambda_{i, r_{i}}}\right)$. Then, $P_{i 12}=V_{i} \tilde{\lambda}_{i}$ and the corresponding matrices $P_{i}$ and $Q_{i}$ are given as

$$
\begin{aligned}
P_{i} & =\left[\begin{array}{cc}
\mathcal{B}_{i} W_{1 i} \mathcal{B}_{i}^{T}+C_{i}^{T} W_{2 i} C_{i} & V_{i} \tilde{\lambda}_{i} \\
\star & I_{r_{i}}
\end{array}\right] \\
Q_{i} & =\left[\begin{array}{cc}
Q_{i 11} & -Q_{i 11} V_{i} \tilde{\lambda}_{i} \\
\star & I_{r_{i}}+\tilde{\lambda}_{i} V_{i}^{T} Q_{i 11} V_{i} \tilde{\lambda}_{i}
\end{array}\right]
\end{aligned}
$$

Hence, matrices $P$ and $Q$ are obtained.

3) Solve the LMI minimization problem in (25) with the derived matrix $P$ in step 2 and derive the dynamic compensator matrix $K=\operatorname{diag}\left(K_{i}\right)$.

4) The matrices $T_{i 1}$ and $T_{i 2}$ in the SMC function (8) are derived with $W_{2 i}$ in step 1 and $P_{i 12}$ in step 2 .

$$
T_{i 1}=B_{i}^{T} C_{i}^{T} W_{2 i}, T_{i 2}=B_{i}^{T} V_{i} \tilde{\lambda}_{i}
$$

Remark 3.2. (i) The orders of the LMI formulations (26)(28) in Corollary 3.2 are less than the formulations (18), (19) in Corollary 3.1. Hence, compared with the multi-step iterative algorithm, the iteration-free algorithm has the advantage of the lower space dimension and computational complexity, which is more conducive to solving the non-convex constraints in (12). (ii) Compared with the FTC based on fault detection and isolation approach[11], [15], the nonlinear SM-FTC switching compensation (13) does not need a fault diagnosis mechanism, and the DFTC protocol is designed by the local output feedbacks without the threshold setting and fault isolation. (iii) Unlike the adaptive approximation algorithm of the unknown disturbance effects and actuator fault function[21], [22], [33], the unknown functions in this study are compensated through adaptively approximating a surrogate of the estimated upper bound parameters. Furthermore, the known boundedness of the disturbances/faults[13], [16], [24], [26] and their first-order derivatives[35] is not required in this study.

Consider the SMC function for the $i$-th agent in (8). The time derivative of the SMC function $\sigma_{i}\left(\bar{x}_{i}, y_{i}, t\right)$ is given as

$$
\begin{aligned}
\dot{\sigma}_{i}\left(\bar{x}_{i}, y_{i}, t\right)= & T_{i} \tilde{C}_{i}\left(\tilde{A}_{i}+\tilde{B}_{i} K_{i} \tilde{C}_{i}\right) \tilde{x}_{i}+T_{i} \tilde{C}_{i} \tilde{F}_{i} f_{i} \\
& +T_{i} \tilde{C}_{i} \tilde{B}_{i 0}\left(d_{i}+v_{i}\right)+T_{i} \tilde{C}_{i} \tilde{G}_{i} \xi_{i}
\end{aligned}
$$

It is necessary to prove the reachability of the sliding surface $\sigma_{i}\left(\bar{x}_{i}, y_{i}, t\right)$ and the following theorem is given.

Theorem 3.2. Consider the pre-calculated matrix $P=$ $\operatorname{diag}\left(P_{i}\right)$ satisfying $(12)$ in Theorem 3.1, the SMC function (8) for each agent (1) can be reached with the adaptive algorithms (9), (10) for the unknown upper bounds and the SM-FTC algorithm (13) in the decentralized control input (4).

Proof. Consider a Lyapunov function $V_{\sigma_{i}}$ for the SMC function $\sigma_{i}\left(\bar{x}_{i}, y_{i}, t\right)$ in $(8)$ as

$$
\begin{aligned}
V_{\sigma_{i}}= & \sum_{i=1}^{N} \sigma_{i}^{T}\left(\bar{x}_{i}, y_{i}, t\right)\left(\tilde{B}_{i 0}^{T} P_{i} \tilde{B}_{i 0}\right)^{-1} \sigma_{i}\left(\bar{x}_{i}, y_{i}, t\right) \\
& +\sum_{i=1}^{N}\left\|P_{i}\right\|\left\|\tilde{C}_{i}\right\|^{-1}\left(e_{d i}^{2}+e_{f i}^{2}\right)
\end{aligned}
$$

The time derivative of $V_{\sigma_{i}}$ in (38) is derived in the following form with a positive scalar $\eta_{i}$.

$$
\begin{aligned}
\dot{V}_{\sigma_{i}}= & 2 \sum_{i=1}^{N} \sigma_{i}^{T}\left(\bar{x}_{i}, y_{i}, t\right)\left(\tilde{B}_{i 0}^{T} P_{i} \tilde{B}_{i 0}\right)^{-1} \\
& \times\left(T_{i} \tilde{\tilde{C}}_{i}\left(\tilde{A}_{i}+\tilde{B}_{i} K_{i} \tilde{C}_{i}\right) \tilde{x}_{i}+T_{i} \tilde{C}_{i} \tilde{B}_{i 0}\left(d_{i}+v_{i}\right)\right. \\
& \left.+T_{i} \tilde{C}_{i} \tilde{F}_{i} f_{i}+T_{i} \tilde{C}_{i} \tilde{G}_{i} \xi_{i}\right) \\
& +\sum_{i=1}^{N} 2\left\|P_{i}\right\|\left\|\tilde{C}_{i}\right\|^{-1}\left\|\tilde{y}_{i}\right\|\left(\left\|B_{i}\right\| e_{d i}+\left\|F_{i}\right\| e_{f i}\right) \\
\leq & \tilde{x}^{T}\left(P \tilde{A}+\tilde{A}^{T} P+P \tilde{B} K \tilde{C}+\tilde{C}^{T} K^{T} \tilde{B}^{T} P\right. \\
& \left.+P P+\tilde{E}^{T} \tilde{E}\right) \tilde{x}-\sum_{i=1}^{N} 2\left\|\tilde{x}_{i}\right\| \eta_{i} \\
& +\sum_{i=1}^{N}\left(2\left\|\tilde{x}_{i}\right\|\left\|P_{i}\right\|\left(\left\|B_{i}\right\| \bar{d}_{i}+\left\|F_{i}\right\| \bar{f}_{i}\right)\right) \\
& +\sum_{i=1}^{N} 2\left\|P_{i}\right\|\left\|\tilde{x}_{i}\right\|\left(\left\|B_{i}\right\| e_{d i}+\left\|F_{i}\right\| e_{f i}\right) \\
& -\sum_{i=1}^{N} 2\left\|\tilde{x}_{i}\right\|\left\|P_{i}\right\|\left(\left\|B_{i}\right\| \hat{\bar{d}}_{i}+\left\|F_{i}\right\| \hat{\bar{f}}_{i}\right) \\
\leq & \tilde{x}^{T}\left(P \tilde{A}+\tilde{A}^{T} P+P \tilde{B}^{T} K \tilde{C}+\tilde{C}^{T} K^{T} \tilde{B}^{T} P\right. \\
& \left.+P P+\tilde{E}^{T} \tilde{E}\right) \tilde{x}-\sum_{i=1}^{N} 2\left\|\tilde{x}_{i}\right\| \eta_{i}
\end{aligned}
$$

It follows that $\dot{V}_{\sigma_{i}}<0$ when the inequality in (12) and $\eta_{i}>0$ are satisfied, and each SMC function $\sigma_{i}\left(\bar{x}_{i}, y_{i}, t\right)=0$ can be reached and subsequently remained there.

Remark 3.3. From Theorems 3.1 and 3.2, both the stability of HMASs and the reachability of the SMC motion depend on the nonlinear inequality constraint in (12): $P \tilde{A}+\tilde{A}^{T} P+$ $P \tilde{B} K \tilde{C}+\tilde{C}^{T} K^{T} \tilde{B}^{T} P+P P+\tilde{E}^{T} \tilde{E}<0$ and $\eta_{i}>0$. The positive scalar $\eta_{i}$ is related with the estimated upper bounds of the matched disturbances and actuator faults $\left(\hat{\bar{d}}_{i}\right.$ and $\left.\hat{\bar{f}}_{i}\right)$ in the SM-FTC function (13). In fact, the SMC function $\sigma_{i}\left(\bar{x}_{i}, y_{i}, t\right)$ is essential to limit the updates in $\hat{\bar{d}}_{i}$ and $\hat{\bar{f}}_{i}$. Furthermore, 
the parameters $\bar{A}_{i}, \bar{B}_{i}, \bar{C}_{i}, \bar{D}_{i}$ in the dynamic compensator $(3),(4)$ and the matrix $T_{i}$ in the SMC function (8) can be derived in the two multi-step algorithms with iteration and iteration-free strategies.

\section{OBSERVER-BASED INTEGRAL SMC DESIGN}

In this section, both the matched disturbances and actuator faults occur in the control input. The observer-based integral SMC design can effectively reject the matched perturbations and improve the robustness of the unmatched interactions.

First, the linear Luenberger observer is designed as

$$
\begin{aligned}
& \dot{\hat{x}}_{i}(t)=A_{i} \hat{x}_{i}(t)+B_{i} u_{i 0}(t)+L_{i}\left(y_{i}(t)-\hat{y}_{i}(t)\right) \\
& \hat{y}_{i}(t)=C_{i} \hat{x}_{i}(t)
\end{aligned}
$$

where $\hat{x}_{i}$ and $\hat{y}_{i}$ are the respective estimations of $x_{i}$ and $y_{i}$. Matrix $L_{i} \in R^{n_{i} \times p_{i}}$ is the observer gain and $u_{i 0}=\mathcal{K}_{i} \hat{x}_{i}$ is the linear component to make the SMC motion stable.

Define the state error $e_{x i}=x_{i}-\hat{x}_{i}$ and the decentralized control input $u_{i}=u_{i 0}+u_{i 1}$ with the nonlinear FTC component $u_{i 1}$ to compensate for the effects of disturbances and actuator faults and to guarantee the system trajectories to remain within the SMC surface. Then, the state error dynamics are given as

$$
\begin{aligned}
\dot{e}_{x i}(t)= & \left(A_{i}-L_{i} C_{i}\right) e_{x i}(t)+\xi_{i}(x, t) \\
& +B_{i}\left(u_{i 1}(t)+d_{i}\left(x_{i}, u_{i}, t\right)+f_{i}(t)\right)
\end{aligned}
$$

The SMC function here is modified in the following compact with an integral component.

$$
\begin{aligned}
\sigma_{i}\left(\hat{x}_{i}, y_{i}, t\right)= & R_{i}\left(y_{i}(t)-y_{i}\left(t_{0}\right)\right) \\
& -R_{i} \int_{t_{0}}^{t} C_{i}\left(A_{i} \hat{x}_{i}(s)+B_{i} u_{i 0}(s)\right) d s
\end{aligned}
$$

where matrix $R_{i}=B_{i}^{T} C_{i}^{\dagger}$ is designed to guarantee the invertibility of matrix $R_{i} C_{i} B_{i}$ and $C_{i}^{\dagger}=\left(C_{i}^{T} C_{i}\right)^{-1} C_{i}^{T}$. The SMC function in (42) implies that the system trajectories start from the SMC manifold, i.e., $\sigma_{i}\left(\hat{x}_{i}, y_{i}, t_{0}\right)=0$. Then, the time derivative of the SMC function (42) is derived as

$$
\begin{aligned}
\dot{\sigma}_{i}\left(\hat{x}_{i}, y_{i}, t\right)= & R_{i} C_{i}\left(A_{i} e_{x i}(t)+\xi_{i}(x, t)\right. \\
& \left.+B_{i}\left(u_{i 1}(t)+d_{i}\left(x_{i}, u_{i}, t\right)+f_{i}(t)\right)\right)
\end{aligned}
$$

Here, an extra bound condition of the nonlinear interaction term is given as $\left\|\xi_{i}(x, t)\right\| \leq \bar{\xi}_{i}$ with the unknown upper bound $\bar{\xi}_{i}$. The parameter estimation error $e_{\xi i}$ is defined as $e_{\xi i}=\hat{\bar{\xi}}_{i}-$ $\bar{\xi}_{i}$ with the estimation of the upper bound $\hat{\bar{\xi}}_{i}$. Furthermore, the adaptive algorithms for the estimated upper bound parameters $\dot{\overline{\hat{d}}}_{i}, \dot{\hat{\bar{f}}}_{i}$ and $\dot{\hat{\bar{\xi}}}_{i}$ are modified as

$$
\begin{gathered}
\dot{\overline{\bar{\xi}}}_{i}=\left\|C_{i}\right\|\left\|\sigma_{i}\left(\hat{x}_{i}, y_{i}, t\right)\right\|, i=1, \cdots, N \\
\dot{\bar{f}}_{i}=\dot{\overline{\hat{d}}}_{i}=\left\|\sigma_{i}\left(\hat{x}_{i}, y_{i}, t\right)\right\|, i=1, \cdots, N
\end{gathered}
$$

Theorem 4.1. Given a matrix $R_{i}=B_{i}^{T} C_{i}^{\dagger}$ which satisfies the invertibility of matrix $R_{i} C_{i} B_{i}$ and a large enough scalar $\bar{\eta}_{i}$, the SMC function (42) can be reached with the adaptive algorithm (44) and the nonlinear FTC input as follows

$$
\begin{aligned}
u_{i 1}(t)= & -\left(\left\|\left(R_{i} C_{i} B_{i}\right)^{-1} R_{i}\right\|\left(\left\|C_{i}\right\| \hat{\bar{\xi}}_{i}\right)\right. \\
& \left.+\hat{\bar{f}}_{i}+\hat{\bar{d}}_{i}+\bar{\eta}_{i}\right) \tanh \left(\sigma_{i}\left(\hat{x}_{i}, y_{i}, t\right)\right)
\end{aligned}
$$

Furthermore, given diagonal matrices $\mathcal{A}$ and $\mathcal{J}$ with elements of the respective $\mathcal{A}_{i}=B_{i} B_{i}^{\dagger} A_{i}$ and $\mathcal{J}_{i}=I_{n_{i}}-B_{i} B_{i}^{\dagger}$, the HMASs are asymptotically stable and the overall state estimation errors $e_{x}=\operatorname{col}\left(e_{x i}\right)$ are ultimately bounded if there exist symmetric positive-definite diagonal matrices $S_{1}$ and $S_{2}$ with elements of matrices $S_{i 1} \in R^{n_{i} \times n_{i}}$ and $S_{i 2} \in R^{n_{i} \times n_{i}}$, and diagonal matrices $L$ and $\mathcal{K}$ with elements of matrices $L_{i} \in R^{n_{i} \times p_{i}}$ and $\mathcal{K}_{i} \in R^{m_{i} \times n_{i}}$ such that

$$
\left[\begin{array}{cccc}
\Phi_{11} & \Phi_{12} & S_{1} \mathcal{J} & S_{2} \mathcal{J} \\
\star & \Phi_{22} & 0 & 0 \\
\star & \star & -I_{n} & 0 \\
\star & \star & \star & -I_{n}
\end{array}\right]<0
$$

with $\Phi_{11}=S_{1} A+A^{T} S_{1}+S_{1} B \mathcal{K}+\mathcal{K}^{T} B^{T} S_{1}+E^{T} E, \Phi_{12}=$ $-S_{1} B \mathcal{K}-S_{1} \mathcal{A}$ and $\Phi_{22}=S_{2} A+A^{T} S_{2}-S_{2} L C-C^{T} L^{T} S_{2}-$ $S_{2} \mathcal{A}-\mathcal{A}^{T} S_{2}+E^{T} E$, where matrices $A, B$ and $C$ are of diagonal forms with elements of $A_{i}, B_{i}$ and $C_{i}$, respectively.

Proof. Consider a Lyapunov function $V_{\hat{\sigma}_{i}}$ for the SMC function $\sigma_{i}\left(\hat{x}_{i}, y_{i}, t\right)$ in $(42)$ as

$$
\begin{aligned}
V_{\hat{\sigma}_{i}}= & \frac{1}{2} \sum_{i=1}^{N}\left(\sigma_{i}^{T}\left(\hat{x}_{i}, y_{i}, t\right)\left(R_{i} C_{i} B_{i}\right)^{-1} \sigma_{i}\left(\hat{x}_{i}, y_{i}, t\right)\right. \\
& \left.+\left\|\left(R_{i} C_{i} B_{i}\right)^{-1} R_{i}\right\| e_{\xi_{i}}^{2}+e_{d_{i}}^{2}+e_{f_{i}}^{2}\right)
\end{aligned}
$$

The time derivative of $V_{\hat{\sigma}_{i}}$ in (47) is obtained as

$$
\begin{aligned}
\dot{V}_{\hat{\sigma}_{i}}= & \sum_{i=1}^{N} \sigma_{i}^{T}\left(\hat{x}_{i}, y_{i}, t\right)\left(R_{i} C_{i} B_{i}\right)^{-1}\left(R_{i} C_{i} A_{i} e_{x i}\right. \\
& \left.+R_{i} C_{i} \xi_{i}\right)+\sum_{i=1}^{N} \sigma_{i}^{T}\left(\hat{x}_{i}, y_{i}, t\right)\left(u_{i 1}+d_{i}+f_{i}\right) \\
& +\sum_{i=1}^{N}\left\|\sigma_{i}\left(\hat{x}_{i}, y_{i}, t\right)\right\|\left(\left\|\left(R_{i} C_{i} B_{i}\right)^{-1} R_{i}\right\|\right. \\
& \left.\times\left(e_{\xi_{i}}\left\|C_{i}\right\|\right)+e_{d_{i}}+e_{f_{i}}\right) \\
\leq & \sum_{i=1}^{N}\left\|\sigma_{i}\left(\hat{x}_{i}, y_{i}, t\right)\right\|\left\|\left(R_{i} C_{i} B_{i}\right)^{-1} R_{i} C_{i} A_{i}\right\|\left\|e_{x i}\right\| \\
& +\sum_{i=1}^{N}\left\|\sigma_{i}\left(\hat{x}_{i}, y_{i}, t\right)\right\|\left(\left\|\left(R_{i} C_{i} B_{i}\right)^{-1} R_{i}\right\|\left\|C_{i}\right\| \bar{\xi}_{i}\right. \\
& \left.+\bar{f}_{i}+\bar{d}_{i}\right)+\sum_{i=1}^{N} \sigma_{i}^{T}\left(\hat{x}_{i}, y_{i}, t\right) u_{i 1} \\
& +\sum_{i=1}^{N}\left\|\sigma_{i}\left(\hat{x}_{i}, y_{i}, t\right)\right\|\left(\left\|\left(R_{i} C_{i} B_{i}\right)^{-1} R_{i}\right\|\right. \\
& \left.\times\left(e_{\xi_{i}}\left\|C_{i}\right\|\right)+e_{d_{i}}+e_{f_{i}}\right) \\
\leq & \sum_{i=1}^{N}\left\|\sigma_{i}\left(\hat{x}_{i}, y_{i}, t\right)\right\| \\
& \times\left(\left\|\left(R_{i} C_{i} B_{i}\right)^{-1} R_{i} C_{i} A_{i}\right\|\left\|e_{x i}\right\|-\bar{\eta}_{i}\right)
\end{aligned}
$$

It is easy to check that the system trajectory will remain within the SMC function if the Euclidean norm of the state estimation error $\left\|e_{x i}\right\|$ is bounded and decreasing. Furthermore, define $\bar{\eta}_{i}=\left\|\left(R_{i} C_{i} B_{i}\right)^{-1} R_{i} C_{i} A_{i}\right\|\left\|e_{x i}\left(t_{0}\right)\right\|+\epsilon_{i}$, where $\epsilon_{i}$ is a positive scalar. Hence, it is derived as

$$
\dot{V}_{\hat{\sigma}_{i}} \leq-\sum_{i=1}^{N} \epsilon_{i}\left\|\sigma_{i}\left(\hat{x}_{i}, y_{i}, t\right)\right\| \leq 0
$$

This implies that the SMC function can be reached with the nonlinear FTC strategy (45) and the reachability analysis is completed. Hence, the dynamic trajectory of HMASs can remain within it on the basis of the stability of the state estimation error $e_{x i}$, which needs to be further dealt with.

Note that the dynamic trajectory can remain within the SMC surface, i.e., $\sigma_{i}\left(\hat{x}_{i}, y_{i}, t\right)=0$ and $\dot{\sigma}_{i}\left(\hat{x}_{i}, y_{i}, t\right)=0$. Hence, the equivalent FTC input $u_{i 1}$ can be derived as

$$
\begin{aligned}
u_{i 1}= & -\left(R_{i} C_{i} B_{i}\right)^{-1}\left(R_{i} C_{i} A_{i} e_{x i}+R_{i} C_{i} B_{i} d_{i}\right. \\
& \left.+R_{i} C_{i} B_{i} f_{i}+R_{i} C_{i} \xi_{i}\right)
\end{aligned}
$$

Then, the dynamics of the $i$-th agent can be modified as

$$
\begin{aligned}
\dot{x}_{i} & =A_{i} x_{i}+B_{i} u_{i 0}-\mathcal{A}_{i} e_{x i}+\mathcal{J}_{i} \xi_{i} \\
& =\left(A_{i}+B_{i} \mathcal{K}_{i}\right) x_{i}-\left(B_{i} \mathcal{K}_{i}+\mathcal{A}_{i}\right) e_{x i}+\mathcal{J}_{i} \xi_{i}
\end{aligned}
$$

where matrices $\mathcal{A}_{i}=B_{i} B_{i}^{\dagger} A_{i}$ and $\mathcal{J}_{i}=I_{n_{i}}-B_{i} B_{i}^{\dagger}$. Note that the matched disturbances $d_{i}$ and actuator faults $f_{i}$ are 
completely compensated with the equivalent FTC input (50). Then, the state estimation error dynamics are also modified as

$$
\dot{e}_{x i}=\left(A_{i}-L_{i} C_{i}-\mathcal{A}_{i}\right) e_{x i}+\mathcal{J}_{i} \xi_{i}
$$

Furthermore, consider a Lyapunov function $V\left(x_{i}, e_{x i}\right)$ for the dynamics (51) and the state estimation error dynamics (52) with symmetric positive-definite matrices $S_{i 1}$ and $S_{i 2}$.

$$
V\left(x_{i}, e_{x i}\right)=\sum_{i=1}^{N}\left(x_{i}^{T} S_{i 1} x_{i}+e_{x i}^{T} S_{i 2} e_{x i}\right)
$$

The time-derivative of $V\left(x_{i}, e_{x i}\right)$ in (53) is obtained as

$$
\begin{aligned}
\dot{V}\left(x_{i}, e_{x i}\right)= & \sum_{i=1}^{N} x_{i}^{T}\left(S_{i 1}\left(A_{i}+B_{i} \mathcal{K}_{i}\right)+\left(A_{i}+B_{i} \mathcal{K}_{i}\right)^{T}\right. \\
& \left.\times S_{i 1}\right) x_{i}-2 \sum_{i=1}^{N} x_{i}^{T} S_{i 1}\left(B_{i} \mathcal{K}_{i}+\mathcal{A}_{i}\right) e_{x i} \\
& +\sum_{i=1}^{N} e_{x i}^{T}\left(S_{i 2}\left(A_{i}-L_{i} C_{i}-\mathcal{A}_{i}\right)\right. \\
& \left.+\left(A_{i}-L_{i} C_{i}-\mathcal{A}_{i}\right)^{T} S_{i 2}\right) e_{x i} \\
& +2 \sum_{i=1}^{N} e_{x i}^{T} S_{i 2} \mathcal{J}_{i} \xi_{i}+2 \sum_{i=1}^{N} x_{i}^{T} S_{i 1} \mathcal{J}_{i} \xi_{i} \\
\leq & {\left[\begin{array}{c}
x \\
e_{x}
\end{array}\right]^{T}\left[\begin{array}{cc}
\tilde{\Phi}_{11} & \tilde{\Phi}_{12} \\
\star & \tilde{\Phi}_{22}
\end{array}\right]\left[\begin{array}{c}
x \\
e_{x}
\end{array}\right] }
\end{aligned}
$$

where $\tilde{\Phi}_{11}=S_{1} A+A^{T} S_{1}+S_{1} B \mathcal{K}+\mathcal{K}^{T} B^{T} S_{1}+E^{T} E+$ $S_{1} \mathcal{J} \mathcal{J}^{T} S_{1}, \tilde{\Phi}_{12}=-S_{1} B \mathcal{K}-S_{1} \mathcal{A}$ and $\tilde{\Phi}_{22}=S_{2} A+A^{T} S_{2}-$ $S_{2} L C-C^{T} L^{T} S_{2}-S_{2} \mathcal{A}-\mathcal{A}^{T} S_{2}+E^{T} E+S_{2} \mathcal{J} \mathcal{J}^{T} S_{2}$.

The inequality in (54) is equivalent to that in (46) with Schur Lemma. Hence, $\dot{V}\left(x_{i}, e_{x i}\right)<0$ is obtained, which implies the asymptotic stability of the dynamic system (51) and the state estimation error (52). This completes the proof.

Remark 4.1. (i) It follows that $V\left(x_{i}, e_{x i}\right)$ is bounded, i.e., $x_{i}$ and $e_{x i}$ are bounded. On the basis of the bounded parameters $x_{i}, e_{x i}$ and $\xi_{i}, \dot{x}_{i}$ and $\dot{e}_{x i}$ are bounded, i.e., $\dot{V}\left(x_{i}, e_{x i}\right)$ is bounded as well. Thus, the condition that the derivative of $V\left(x_{i}, e_{x i}\right)$ in (53) should be uniformly continuous with respect to $t$ is satisfied. (ii) Since $\dot{V}\left(x_{i}, e_{x i}\right)<0$ in (54) and the uniform continuity of the derivative of $V\left(x_{i}, e_{x i}\right)$ in (53), it follows that $\lim _{t \rightarrow \infty} \dot{V}\left(x_{i}, e_{x i}\right)=0,\left\|x_{i}\right\| \rightarrow 0$ and $\left\|e_{x i}\right\| \rightarrow 0$ are satisfied by using Barbalat Lemma.

Remark 4.2. The conventional separation principle cannot be used in HMASs because of the existing interactions among HMASs. The bidirectional interaction term $\xi_{i}(x, t)$ appears in both the state dynamics (51) and the estimation error dynamics (52). In comparison with the work that the interactions are satisfied with the matching condition[24], [32], the unmatched interactions in this study are further handled with additional freedoms provided in the integral SMC design.

Remark 4.3. (i) The decentralized controller in the integral SMC contains two parts, namely, the linear and nonlinear FTC compensating components. The nominal linear controller serves to guarantee the stability of the SMC motion, whereas the FTC compensating controller aims to attenuate the effect caused by disturbances and actuator faults. (ii) As opposed to the discontinuous dynamics in the SMC protocol[33] and the sign function $\operatorname{sgn}(\cdot)$ in (13), the nonlinear FTC input in the integral SMC protocol is continuous within a boundary layer of the hyperbolic tangent $\tanh (\cdot)$ and prevents system chattering[30], [31]. More importantly with the help of an adaptive mechanism, the corresponding protocol can work effectively even without prior knowledge of the actuator faults and interactions[13], [21], [22], [25].

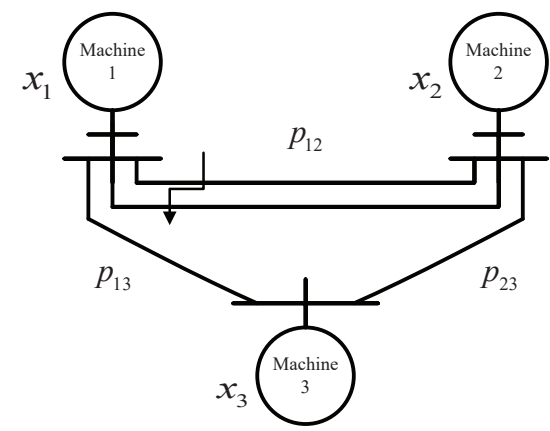

Fig. 1. The 3-machine power systems.

TABLE I

THE PHYSICAL MEANINGS AND VALUES OF THE PARAMETERS

\begin{tabular}{|c|c|c|c|c|}
\hline Parameter & Physical meaning & M1 & M2 & M3 \\
\hline$D$ & Damping coefficient & 5 & 3 & 3 \\
\hline$H$ & Inertia time constant & 4 & 5.1 & 5.1 \\
\hline$\omega_{o}$ & Synchronous speed & 314.2 & 314.2 & 314.2 \\
\hline$T_{m}$ & Time constant of turbine & 0.35 & 0.35 & 0.35 \\
\hline$T_{e}$ & Time constant of speed governor & 0.1 & 0.1 & 0.1 \\
\hline$K_{m}$ & Gain of turbine & 1 & 1 & 1 \\
\hline$K_{e}$ & Gain of speed governor & 1 & 1 & 1 \\
\hline$R$ & Regulation constant & 0.05 & 0.05 & 0.05 \\
\hline \multicolumn{5}{|r}{} \\
\end{tabular}

\section{Simulation RESULTS}

In this section, a numerical simulation of 3-machine power systems with nonlinear interactions and actuator faults is put forward to validate the effectiveness of the proposed control designs, i.e., the decentralized output SM-FTC scheme and the observer-based integral SMC scheme.

The HMASs model of the 3-machine power systems with steam valve control is given in the form of (1) with the state vector $x_{i}=\left[\begin{array}{lll}\Delta \delta_{i}^{T} & \omega_{i}^{T} & \Delta P_{m i}^{T} \Delta X_{e i}^{T}\end{array}\right]^{T}$ of each machine, where $\Delta \delta_{i}=\delta_{i}-\delta_{i 0}, \Delta P_{m i}=P_{m i}-P_{m i 0}$ and $\Delta X_{e i}=X_{e i}-X_{e i 0}$. $\delta_{i}, \omega_{i}, P_{m i}$ and $X_{e i}$ denote the rotor angle, relative speed, per unit mechanical power and steam valve aperture of the $i$-th machine $(i=1,2,3)$, respectively. $\delta_{i 0}, P_{m i 0}$ and $X_{e i 0}$ denote the respective nominal values. Furthermore, the 3-machine power systems are characterized by the following matrices[36].

$$
A_{i}=\left[\begin{array}{cccc}
0 & 1 & 0 & 0 \\
0 & -\frac{D_{i}}{2 H_{i}} & \frac{\omega_{o}}{2 H_{i}} & 0 \\
0 & 0 & -\frac{1}{T_{m i}} & \frac{K_{m i}}{T_{m_{i}}} \\
0 & -\frac{K_{e i}}{T_{e i} R_{i} \omega_{o}} & 0 & -\frac{1}{T_{e i}}
\end{array}\right], B_{i}=\left[\begin{array}{c}
0 \\
0 \\
0 \\
\frac{1}{T_{e i}}
\end{array}\right]
$$

The structure of the 3-machine power systems is shown in Figure 1 and the physical meanings and values of the parameters are illustrated in TABLE I[36], [37]. Furthermore, the nonlinear interaction term $\xi_{i}(x, t)$ is given as

$$
\xi_{i}(x, u, t)=\sum_{i=1}^{3} p_{i j}\left[0-\frac{\omega_{o} q_{i} q_{j} B_{i j}}{2 H_{i}} 00\right]^{T} \sin \left(\Delta \delta_{i}-\Delta \delta_{j}\right)
$$

where $p_{i j}$ is a weight coefficient and $p_{i i}=0, p_{i j}=1$ if there is a connection between the $i$-th and $j$-th machines, otherwise $p_{i j}=0, q_{i}$ and $q_{j}$ are the per unit internal transient voltages and $B_{i j}$ is per unit nodal susceptance between the $i$-th and $j$-th 
machines. It follows that the overall interaction term is satisfied with $\xi^{T}(x, t) \xi(x, t) \leq x^{T}\left(L_{\max }^{T} \otimes C_{m}^{T}\right)\left(L_{\max } \otimes C_{m}\right) x$.

$L_{\max }=\left[\begin{array}{ccc}0.6598 & -0.3299 & -0.3299 \\ -0.2772 & 0.5544 & -0.2772 \\ -0.3299 & -0.2772 & 0.6071\end{array}\right], C_{m}=\left[\begin{array}{ll}1 & 0 \\ 1 \times 3\end{array}\right]$

To demonstrate the efficiency of the proposed algorithms in Theorems 3.1, 3.2 and 4.1, the actuator faults in the power systems are considered in the steam valve control inputs and the fault distribution matrices are satisfied with $F_{i}=B_{i}$.

$$
\begin{aligned}
& f_{1}=\left\{\begin{array}{l}
|0.1 \sin (0.5 t)|, t \leq 8 \\
0.05 \operatorname{sat}(0.1 \sin (0.5 t)), t>8
\end{array} \quad, f_{2}=\left\{\begin{array}{l}
0.2, t \leq 40 \\
0.1, t>40
\end{array}\right.\right. \\
& f_{3}=\left\{\begin{array}{l}
|0.2 \sin (0.5 t)|, t \leq 20 \\
0.1 \operatorname{sat}(0.2 \sin (0.5 t)), t>20
\end{array}\right.
\end{aligned}
$$

The matched disturbances $d_{i}, i=1,2,3$ are considered as $d_{1}=0.1 \sin (0.5 t), d_{2}=0.1 \cos (0.5 t)$ and $d_{3}=|0.1 \sin (0.5 t)|$.

Simulation parameters are designed as $\varepsilon=0.01$ and $\mu_{\tilde{C}}=$ $\mu_{W}=0.1$, and the gains in the augmented dynamics (5) and the SMC function (8) are derived by solving Algorithm 3.1.

$$
\begin{aligned}
& T_{1}=\left[\begin{array}{ll}
-5.7768 & 0
\end{array}\right], T_{2}=\left[\begin{array}{ll}
-0.0828 & 0
\end{array}\right], T_{3}=\left[\begin{array}{ll}
-3.7063 & 0
\end{array}\right] \\
& K_{1}=\left[\begin{array}{cc}
-2.2451 & 0.0000 \\
0.0000 & -4.0499
\end{array}\right], K_{2}=\left[\begin{array}{cc}
-1.2882 & 0.0000 \\
0.0000 & -4.0499
\end{array}\right] \\
& K_{3}=\left[\begin{array}{cc}
-1.9541 & 0.0000 \\
0.0000 & -4.0499
\end{array}\right]
\end{aligned}
$$

Furthermore, the following gains, i.e., the symmetric positive definite matrices $P_{i}$ in (12), the SMC matrices $T_{i}$ in (8) and the dynamic compensator matrices $K_{i}$ in (5) are derived by solving Algorithm 3.2.

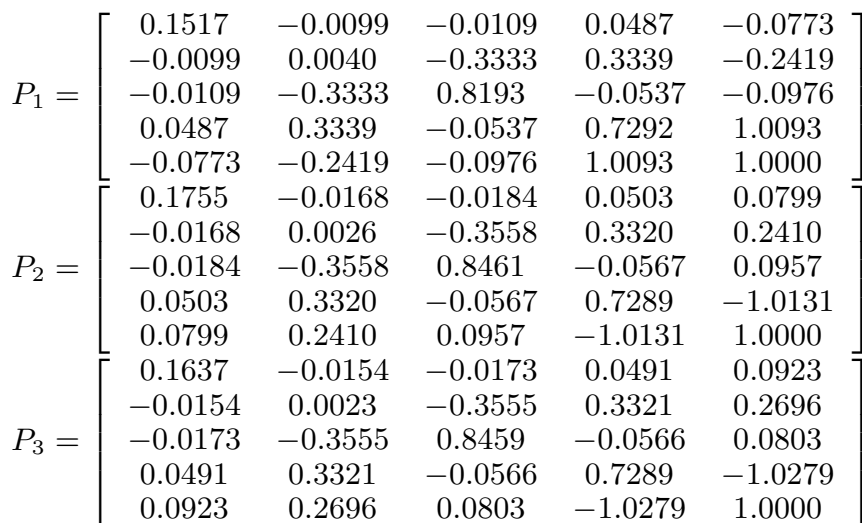

$$
\begin{aligned}
& T_{11}=8.4265, T_{21}=8.1593, T_{31}=8.1774 \\
& T_{12}=10.0931, T_{22}=-10.1309, T_{32}=-10.2794 \\
& K_{1}=\left[\begin{array}{cc}
0.2866 & -1.6224 \\
-1.6224 & 5.6023
\end{array}\right], K_{2}=\left[\begin{array}{ll}
0.3101 & 1.5162 \\
1.5162 & 4.5578
\end{array}\right] \\
& K_{3}=\left[\begin{array}{ll}
0.3314 & 1.5393 \\
1.5393 & 4.6515
\end{array}\right]
\end{aligned}
$$

In the presence of the time-varying additive faults both in the first and third machines and the time-invariant additive faults in the second machine, the results in Figures 2-3 indicate the effectiveness of the decentralized output SM-FTC design in Theorems 3.1 and 3.2. The respective deviations of the rotor angles $\Delta \delta_{i}$ in the three machines are depicted in Figure 2 with the application of Algorithms 3.1 and 3.2. The respective deviations of the relative speed $\omega_{i}$, the per unit mechanical power $\Delta P_{m i}$ and the steam valve aperture $\Delta X_{e i}$ in the three machines in Figure 3 show the robust stability of the 3machine power systems. Note that the first machine fails at $t=8 \mathrm{~s}$, the second one fails at $t=40 \mathrm{~s}$ and the third one suffers a failure at $t=20 \mathrm{~s}$. These figures show that with the proposed SM-FTC strategy, the HMASs are insensitive to the considered actuator faults and the matched disturbances can be compensated in finite-time simulations.
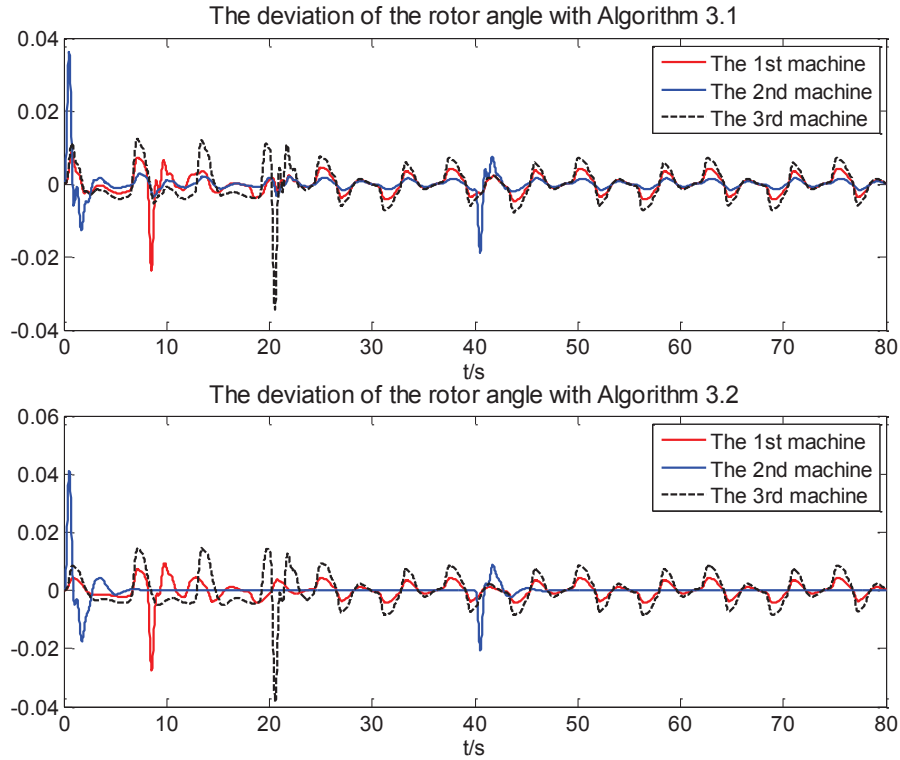

Fig. 2. The respective deviations of the rotor angles $\Delta \delta_{i}$ in three machines with the decentralized output SM-FTC scheme (Algorithms 3.1 and 3.2).
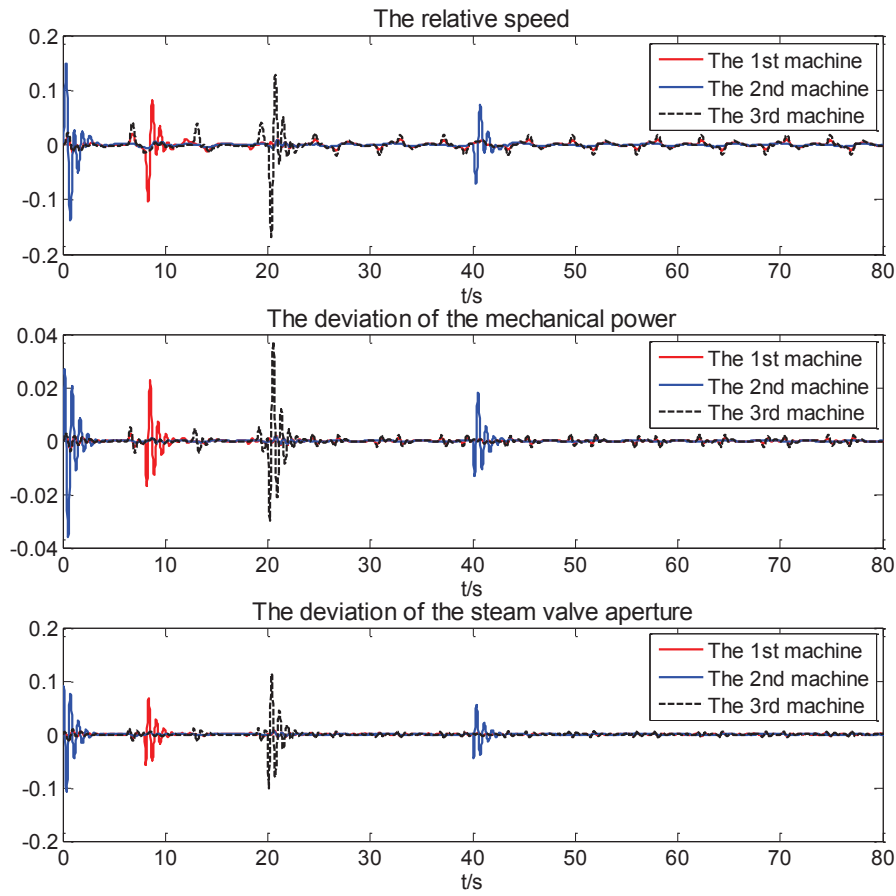

Fig. 3. The respective deviations $\omega_{i}, \Delta P_{m i}$ and $\Delta X_{e i}$ in three machines with the decentralized output SM-FTC scheme.

In the observer-based integral SMC scheme, the following gains, i.e., the linear feedback gains $\mathcal{K}_{i}$ and the observer gains 
$L_{i}$ in (40) are derived by solving Theorem 4.1.

$$
\begin{aligned}
& \mathcal{K}_{1}=\left[\begin{array}{llll}
0.0106 & -0.1521 & -0.0004 & 0.0419
\end{array}\right. \\
& \mathcal{K}_{2}=\left[\begin{array}{llll}
0.0101 & -0.1251 & -0.0008 & 0.0429
\end{array}\right] \\
& \mathcal{K}_{3}=\left[\begin{array}{llll}
0.0101 & -0.1251 & -0.0009 & 0.0429
\end{array}\right] \\
& L_{1}=\left[\begin{array}{c}
-0.3247 \\
3.3132 \\
1.1008 \\
1.7007
\end{array}\right], L_{2}=\left[\begin{array}{c}
-0.2961 \\
3.0191 \\
1.1828 \\
1.6974
\end{array}\right], L_{3}=\left[\begin{array}{c}
-0.2956 \\
3.0177 \\
1.1830 \\
1.6967
\end{array}\right]
\end{aligned}
$$

In the presence of the same actuator faults in the steam valve aperture channel, the results in Figures 4-6 show the effectiveness of the observer-based integral SMC design. Using the proposed control scheme in Theorem 4.1, the time responses of the states in 3-machine power systems are shown in Figures 4-6. These figures indicate that the 3-machine power systems are stabilized and insensitive toward the matched disturbances and faults under the action of actuators in their respective time constants $t=8 \mathrm{~s}, 20 \mathrm{~s}$ and $40 \mathrm{~s}$. The curves in Figure 7 depict the state estimation errors and indicate the good tracking properties of the rated and estimated states of the 3-machine power systems. Only the state estimation errors $e_{x 14}, e_{x 24}$ and $e_{x 34}$ in the fourth channel in three machines show the fluctuations at each fault occurring time instants. The state estimation curves are within the rated values and exhibit small oscillation amplitudes during convergence at each faultoccurring time instance.

Hence, the numerical simulation case demonstrates the effectiveness of the proposed decentralized output SM-FTC and the observer-based integral SMC schemes for the 3-machine power systems.
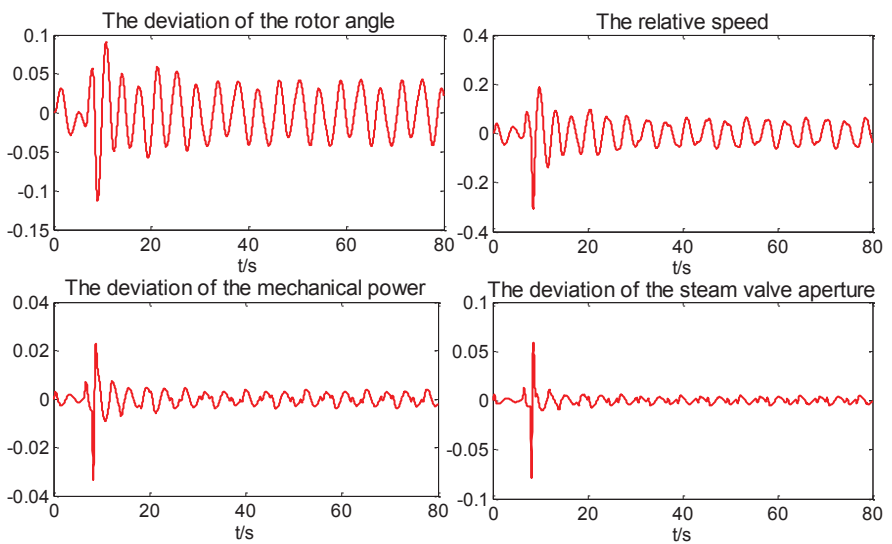

Fig. 4. The respective deviations $\Delta \delta_{i}, \omega_{i}, \Delta P_{m i}$ and $\Delta X_{e i}$ in the first machine with the observer-based integral SMC scheme.

\section{CONCLUSIONS}

In this study, a decentralized output SMC-FTC design is developed for a class of nonlinear HMASs in the presence of matched disturbances, unmatched interactions, and actuator faults. The nonlinear interaction term is treated as a quadratic constraint. Meanwhile, the disturbances and faults are compensated by adaptively estimating the unknown upper bounds. Subsequently, two LMI minimization algorithms, namely, iteration and iteration-free protocols, are integrated into the SM-FTC design to solve the nonlinear matrix inequality.
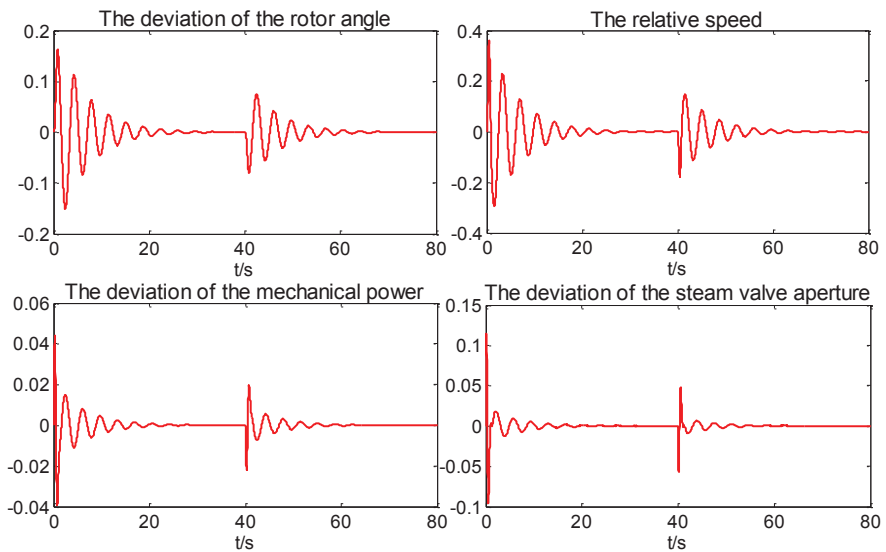

Fig. 5. The deviations $\Delta \delta_{i}, \omega_{i}, \Delta P_{m i}$ and $\Delta X_{e i}$ in the second machine with the observer-based integral SMC scheme.
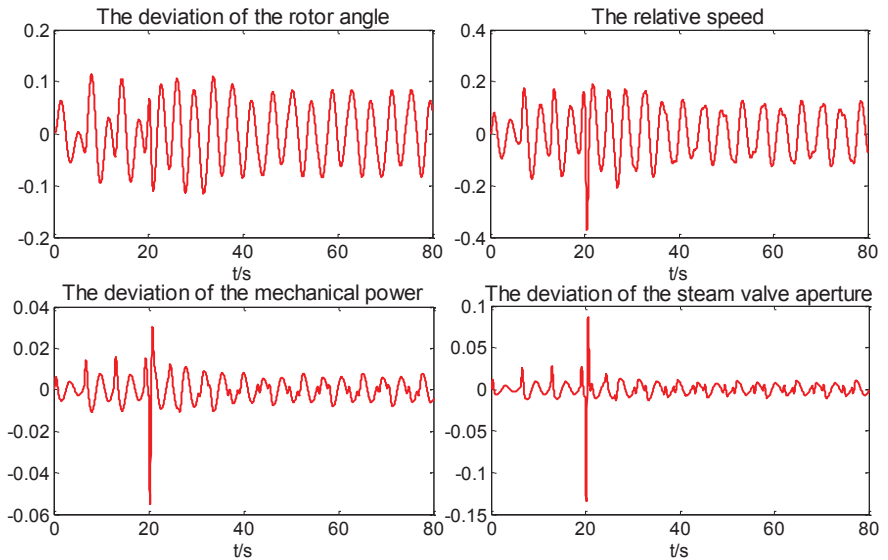

Fig. 6. The respective deviations $\Delta \delta_{i}, \omega_{i}, \Delta P_{m i}$ and $\Delta X_{e i}$ in the third machine with the observer-based integral SMC scheme.

The observer-based integral SMC is further introduced into the proposed SM-FTC scheme to guarantee the asymptotic stability of HMASs and ultimately realize the boundedness of the estimation errors. Current investigations focus on the extension of the proposed method to nonlinear HMASs with model uncertainties, unmatched disturbances and simultaneous actuator/sensor faults under the circumstance of network disconnections and cyber attacks.

\section{REFERENCES}

[1] T. Morstyn, B. Hredzak, and V. G. Agelidis, "Cooperative multi-agent control of heterogeneous storage devices distributed in a DC microgrid," IEEE Trans. Power Syst., vol. 31, no. 4, pp. 2974-2986, Jul. 2016.

[2] H. Bagci, I. Korpeoglu, and A. Yazici, "A distributed fault-tolerant topology control algorithm for heterogeneous wireless sensor networks," IEEE Trans. Parallel Distrib. Syst., vol. 26, no. 4, pp. 914-923, Apr. 2015.

[3] F. A. Yaghmaie, F. L. Lewis, and R. Su, "Output regulation of linear heterogeneous multi-agent systems via output and state feedback," Automatica, vol. 67, pp. 157-164, May. 2016.

[4] C. Huang and X. D. Ye, "Cooperative output regulation of heterogeneous multi-agent systems: an $H_{\infty}$ criterion," IEEE Trans. Autom. Control, vol. 59, no. 1, pp. 267-273, Jan. 2014.

[5] J. Han, H. G. Zhang, H. Jiang, and X. Sun, " $H_{\infty}$ consensus for linear heterogeneous multi-agent systems with state and output feedback control," Neurocomputing, vol. 275, pp. 2635-26944, Jan. 2018. 

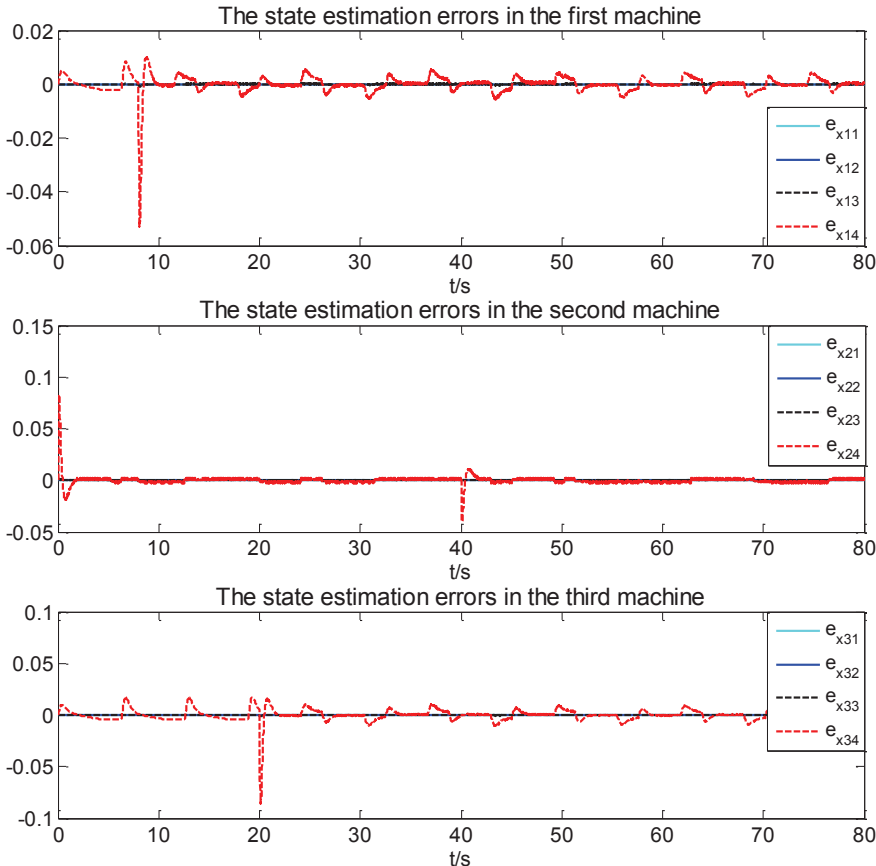

Fig. 7. The respective state estimation errors with the observer-based integral SMC scheme.

[6] Q. Song, F. Liu, G. H. Wen, J. D. Cao, and X. S. Yang, "Distributed position-based consensus of second-order multiagent systems with continuous/intermittent communication," IEEE Trans. Cybern., vol. 47, no. 8, pp. 1860-1871, Aug. 2017.

[7] D. P. Yang, W. Ren, X. D. Liu, and W. S. Chen, "Decentralized eventtriggered consensus for linear multi-agent systems under general directed graphs," Automatica, vol. 69, pp. 242-249, Jul. 2016.

[8] S. C. Tong, L. L. Zhang, and Y. M. Li, "Observed-based adaptive fuzzy decentralized tracking control for switched uncertain nonlinear large-scale systems with dead zones," IEEE Trans. Syst. Man Cybern. Syst., vol. 46, no. 1, pp. 37-47, Jan. 2016.

[9] T. Bian, Y. Jiang, and Z. P. Jiang, "Decentralized adaptive optimal control of large-scale systems with application to power systems," IEEE Trans. Ind. Electron., vol. 62, no. 4, pp. 2439-2447, Apr. 2015.

[10] D. R. Liu, D. Wang, and H. L. Li, "Decentralized stabilization for a class of continuous-time nonlinear interconnected systems using online learning optimal control approach," IEEE Trans. Neural Networks Learning Syst., vol. 25, no. 2, pp. 418-428, Feb. 2014.

[11] M. R. Davoodi, K. Khorasani, H. A. Talebi, and H. R. Momeni, "Distributed fault detection and isolation filter design for a network of heterogeneous multiagent systems," IEEE Trans. Control Syst. Technol., vol. 22, no. 3, pp. 1061-1069, May. 2014.

[12] X. Jin, "Nonrepetitive leader-follower formation tracking for multiagent systems with LOS range and angle constraints using iterative learning control," IEEE Trans. Cybern., to be published.

[13] H. J. Ma and G. H. Yang, "Adaptive fault tolerant control of cooperative heterogeneous systems with actuator faults and unreliable interconnections," IEEE Trans. Autom. Control, vol. 61, no. 11, pp. 3240-3255, Nov. 2016.

[14] M. Saska, T. Krajnik, V. Vonasek, Z. Kasl, V. Spurny, and L. Preucil, "Fault-tolerant formation driving mechanism designed for heterogeneous MAVs-UGVs groups," J. Intell. Rob. Syst., vol. 73, pp. 603-622, Jan. 2014.

[15] M. P. Fanti, A. M. Mangini, and W. Ukovich, "Fault detection by labeled petri nets in centralized and distributed approaches," IEEE Trans. Autom. Sci. Eng., vol. 10, no. 2, pp. 392-404, Apr. 2013.

[16] X. Y. Wang, S. H. Li, and P. Shi, "Distributed finite-time containment control for double-integrator multiagent systems," IEEE Trans. Cybern., vol. 44, no. 9, pp. 1518-1528, Sep. 2014.

[17] H. W. Wang, X. F. Liao, T. W. Huang, and C. J. Li, "Cooperative distributed optimization in multiagent networks with delays," IEEE Trans. Syst. Man Cybern. Syst., vol. 45, no. 2, pp. 363-369, Feb. 2015.
[18] H. Y. Liu, L. Cheng, M. Tan, Z. G. Hou, and Y. P. Wang, "Distributed exponential finite-time coordination of multi-agent systems: containment control and consensus," Int. J. Control, vol. 88, no. 2, pp. 237-247, 2015.

[19] Z. Q. Zuo, J. Zhang, and Y. J. Wang, "Adaptive fault tolerant tracking control for linear and Lipschitz nonlinear multi-agent systems," IEEE Trans. Ind. Electron., vol. 62, no. 6, pp. 3923-3931, Jun. 2015.

[20] S. Shao, H. Yang, B. Jiang, and S. Y. Cheng, "Decentralized fault tolerant control for a class of interconnected nonlinear systems," IEEE Trans. Cybern., vol. 48, no. 1, pp. 178-186, Jan. 2018.

[21] P. Panagi and M. M. Polycarpou, "Decentralized fault tolerant control of a class of interconnected nonlinear systems," IEEE Trans. Autom. Control, vol. 56, no. 1, pp. 178-184, Jan. 2011.

[22] S. J. Yoo, "Neural-network-based decentralized fault-tolerant control for a class of nonlinear large-scale systems with unknown time-delayed interaction faults," J. Franklin Inst., vol. 351, no. 3, pp. 1615-1629, Mar. 2014.

[23] S. C. Tong, B. Y. Huo, and Y. M. Li, "Observer-based adaptive decentralized fuzzy fault-tolerant control of nonlinear large-scale systems with actuator failures," IEEE Trans. Fuzzy Syst., vol. 22, no. 1, pp. 1-15, Feb. 2014.

[24] C. Deng and G. H. Yang, "Decentralized fault-tolerant control for a class of nonlinear large-scale systems with actuator faults," Inf. Sci., vol. 382-383, pp. 334-349, Mar. 2017.

[25] Y. M. Li and S. C. Tong, "Adaptive neural networks decentralized FTC design for nonstrict-feedback nonlinear interconnected large-scale systems against actuator faults," IEEE Trans. Neural Networks Learning Syst., vol. 28, no. 11, pp. 2541-2554, Nov. 2017.

[26] X. J. Li and G. H. Yang, "Neural-network-based adaptive decentralized fault-tolerant control for a class of interconnected nonlinear systems," IEEE Trans. Neural Networks Learning Syst., vol. 29, no. 1, pp. 144155, Jan. 2018.

[27] C. C. Hua, Y. F. Li, H. B. Wang, and X. P. Guan, "Decentralised fault-tolerant finite-time control for a class of interconnected non-linear systems," IET Control Theory Appl., vol. 9, no. 16, pp. 2331-2339, Oct. 2015.

[28] X. Jin, "Adaptive decentralized finite-time output tracking control for MIMO interconnected nonlinear systems with output constraints and actuator faults," Int. J. Robust Nonlinear Control, vol. 28, no. 5, pp. 1808-1829, Mar. 2018.

[29] J. Zhang, M. Lyu, T. F. Shen, L. Liu, and Y. M. Bo, "Sliding mode control for a class of nonlinear multi-agent system with time delay and uncertainties," IEEE Trans. Ind. Electron., vol. 65, no. 1, pp. 865-875, Jan. 2018.

[30] J. H. Qin, G. S. Zhang, W. X. Zheng, and Y. Kang, "Adaptive sliding mode consensus tracking for second-order nonlinear multiagent systems with actuator faults," IEEE Trans. Cybern., vol. PP, no. 99, pp. 1-11, Apr. 2018.

[31] S. Mondal, R. Su, and L. H. Xie, "Heterogeneous consensus of highorder multiagent systems with mismatched uncertainties using sliding mode control," Int. J. Robust Nonlinear Control, vol. 27, no. 13, pp. 2303-2320, Sep. 2017.

[32] Z. K. Li, Z. S. Duan, and F. L. Lewis, "Distributed robust consensus control of multi-agent systems with heterogeneous matching uncertainties," Automatica, vol. 50, no. 3, pp. 883-889, Mar. 2014.

[33] S. H. Yu and X. J. Long, "Finite-time consensus for second-order multiagent systems with disturbances by integral sliding mode," Automatica, vol. 54, pp. 158-165, Apr. 2015.

[34] B. X. Mu, K. W. Zhang, and Y. Shi, "Integral sliding mode flight controller design for a quadrotor and the application in a heterogeneous multi-agent system," IEEE Trans. Ind. Electron., vol. 64, no. 12, pp. 93899398, Dec. 2017.

[35] J. H. Qin, Q. C. Ma, H. J. Gao, and W. X. Zheng, "Fault-tolerant cooperative tracking control via integral sliding mode control technique," IEEE/ASME Trans. Mechatron., vol. 23, no. 1, pp. 342-351, Feb. 2018.

[36] Z. Huang and R. J. Patton, "Output feedback sliding mode FTC for a class of nonlinear inter-connected systems," IFAC-PapersOnLine, vol. 48, no. 21, pp. 1140-1145, 2015.

[37] Z. Huang, R. J. Patton, and J. L. Lan, "Sliding mode state and fault estimation for decentralized systems," in Variable-Structure Approaches, A. Rauh and L. Senkel, Cham: Springer, 2016, pp. 243-281.

[38] P. Shi and Q. K. Shen, "Observer-based leader-following consensus of uncertain nonlinear multi-agent systems," Int. J. Robust Nonlinear Control, vol. 27, no. 17, pp. 3794-3811, Nov. 2017.

[39] D. R. Ding, Z. D. Wang, D. W. C. Ho, and G. L. Wei, "Observerbased event-triggering consensus control for multiagent systems with lossy sensors and cyber-attacks," IEEE Trans. Cybern., vol. 47, no. 8, pp. 1936-1947, Aug. 2017. 
[40] J. Q. Hu, J. D. Cao, J. Yu, and T. Hayat, "Consensus of nonlinear multiagent systems with observer-based protocols," Syst. Control Lett., vol. 72, pp. 71-79, Oct. 2014

[41] B. Zhang, Y. M. Jia, and F. Matsuno, "Finite-time observers for multiagent systems without velocity measurements and with input saturations," Syst. Control Lett., vol. 68, pp. 86-94, Jun. 2014.

[42] S. K. Bag, S. K. Spurgeon, and C. Edwards, "Output feedback sliding mode design for linear uncertain systems," IET Control Theory Appl., vol. 144, no. 3, pp. 209-216, May. 1997.

[43] S. W. Mei and K. Z. Liu, Modern Robust Control Theory and Application. Beijing: Tsinghua University Press, 2003, pp. 84-114.

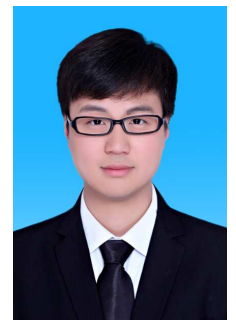

Chun Liu received the B.S. degree in automation and the M.S. degree in control theory and control engineering from the Nanjing University of Aeronautics and Astronautics, Nanjing, China, in 2013 and 2016, respectively, where he is currently pursuing the Ph.D. degree with the College of Automation Engineering.

His current research interests include fault diagnosis and fault tolerant control for multi-agent systems and their applications.

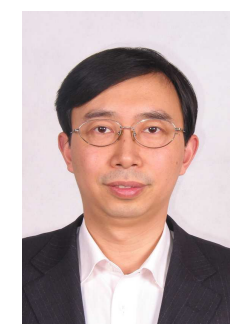

Bin Jiang (SM'05) received the Ph.D. degree in automatic control from Northeastern University, Shenyang, China, in 1995.

He had ever been a Post-Doctoral Fellow, a Research Fellow, an Invited Professor, and a Visiting Professor in Singapore, France, USA and Canada, respectively. He is currently Chair Professor of Cheung Kong Scholar Program with the Ministry of Education and the Vice President of Nanjing University of Aeronautics and Astronautics, Nanjing, China. He has authored eight books and over 200 referred international journal papers and conference papers. His current research interests include intelligent fault diagnosis and fault tolerant control and their applications to helicopters, satellites and high-speed trains.

Dr. Jiang was a recipient of the Second Class Prize of National Natural Science Award of China in 2018. He currently serves as an Associate Editor or an Editorial Board Member for a number of journals, such as the IEEE Transactions on Control Systems Technology, International Journal of Control, Automation and Systems, Journal of Astronautics, Control and Decision, and Systems Engineering and Electronics Technologies. He is a Chair of Control Systems Chapter in IEEE Nanjing Section, a member of IFAC Technical Committee on Fault Detection, Supervision, and Safety of Technical Processes. He has been a Principle Investigator on several projects of National Natural Science Foundation of China.

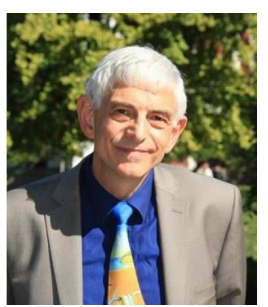

Ron J. Patton (LF'10) received the B.Eng., M.Eng., and Ph.D. degrees in electrical and electronic engineering and control systems from the University of Sheffield, Sheffield, U.K., in 1971, 1974, and 1980, respectively.

$\mathrm{He}$ is currently the Chair of Control and Intelligent Systems Engineering, Hull University, Hull, U.K. He has made a substantial contribution in the field of modeling and design of robust methods for fault detection and isolation and fault tolerant control (FTC) in dynamic systems as the author of 376 papers, including 138 journal papers and six books. His research interests include robust, multiple-model and decentralized control strategies for FTC systems and he has a growing interest in FTC methods for renewable energy. $\mathrm{He}$ is the Senior Member of AIAA and the Fellow of the Institute of Measurement and Control.

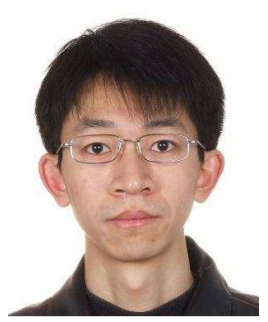

Ke Zhang (SM'17) received the Ph.D. degree in control theory and engineering from the Nanjing University of Aeronautics and Astronautics, Nanjing, China, in 2012.

$\mathrm{He}$ is currently an Associate Professor with the Nanjing University of Aeronautics and Astronautics. His research interests include fault diagnosis and fault tolerant control for dynamical systems and their applications. 